\title{
From complex to simple : hierarchical free-energy landscape renormalized in deep neural networks
}

\author{
Hajime Yoshino ${ }^{1,2 *}$ \\ 1 Cybermedia Center, Osaka University, Toyonaka, Osaka 560-0043, Japan \\ 2 Graduate School of Science, Osaka University, Toyonaka, Osaka 560-0043, Japan \\ *yoshino@cmc.osaka-u.ac.jp
}

October 23, 2019

\begin{abstract}
We develop a statistical mechanical approach based on the replica method to study the solution space of deep neural networks. Specifically we analyze the configuration space of the synaptic weights in a simple feed-forward perceptron network within a Gaussian approximation for two scenarios : a setting with random inputs/outputs and a teacher-student setting. By increasing the strength of constraints, i. e. increasing the number of imposed patterns, successive 2nd order glass transition (random inputs/outputs) or 2nd order crystalline transition (teacher-student setting) take place place layer-by-layer starting next to the inputs/outputs boundaries going deeper into the bulk. In deep enough networks the central part of the network remains in the liquid phase. We argue that in systems of finite width, weak bias field remains in the central part and plays the role of a symmetry breaking field which connects the opposite sides of the system. In the setting with random inputs/outputs, the successive glass transitions bring about a hierarchical free-energy landscape with ultra-metricity, which evolves in space: it is most complex close to the boundaries but becomes renormalized into progressively simpler one in deeper layers. These observations provide clues to understand why deep neural networks operate efficiently. Finally we present results of a set of numerical simulations to examine the theoretical predictions.
\end{abstract}

\section{Contents}

1 Introduction $\quad 3$

2 Model $\quad 5$

2.1 Multi-layer feed-forward network 5

2.2 Two scenarios for inputs/output patterns $\quad 7$

2.2.1 Random inputs/outputs

$\begin{array}{lll}2.2 .2 & \text { Teacher-student setting } & 7\end{array}$

2.3 Modified model $\quad 8$

3 Replica theory $\quad 8$

3.1 Order parameters 8 
3.2 Replicated Gardner volume 9

3.3 Parisi's ansatz $\quad 11$

3.3.1 Random inputs/outputs 11

3.3.2 Teacher-student setting 12

4 Random inputs/outputs $\quad 13$

4.1 Quenched boundary 13

4.1.1 Liquid phase 13

$\begin{array}{lll}4.1 .2 & 1 \text { st glass transition } & 14\end{array}$

$\begin{array}{ll}4.1 .3 & \text { 2nd glass transition } \\ & 15\end{array}$

4.1.4 More glass transitions 16

$\begin{array}{lll}4.2 & \text { Fluctuating boundary } & 19\end{array}$

4.2.1 One RSB type boundary 20

$\begin{array}{lll}4.2 .2 & \text { Full RSB type boundary } 20\end{array}$

5 Teacher-student setting $\quad 21$

5.1 Training 22

5.2 Generalization 23

6 Simulations of learning $\quad 24$

6.1 Relaxational dynamics of a soft-core model with random inputs/outputs $\quad 24$

6.2 Learning by a binary perceptron in a teacher-student setting 26

7 Conclusion and outlook $\quad 28$

$\begin{array}{lll}7.1 & \text { Conclusion } & 28\end{array}$

$\begin{array}{lll}7.2 \text { Outlook } & 29\end{array}$

$\begin{array}{ll}\text { References } & 33\end{array}$

A Replicated free-energy $\quad 36$

A.1 Evaluation of the entropic part of the free-energy 36

A.1.1 Entropic part of the 'bonds' 37

A.1.2 Entropic part of the 'spins' 37

A.2 Evaluation of interaction part of the free-energy 37

$\begin{array}{lll}\text { A.2.1 Original model } & 38\end{array}$

A.2.2 Modified model 39

$\begin{array}{lll}\text { A.3 Total free-energy } & 39\end{array}$

B RSB solution for the random inputs/outputs $\quad 40$

B.1 Entropic part of the free-energy 40

B.1.1 Entropic part of the free-energy due to 'bonds' 40

B.1.2 Entropic part of the free-energy due to 'spins' 41

B.2 Interaction part of the free-energy 43

B.3 Saddle point equations $\quad 43$

B.3.1 Variation of $q_{i}(l)$ 's $\quad 43$

B.3.2 Variation of $G_{i}(l)$ 's $\quad 45$

B.3.3 Procedure to solve the saddle point equations 45 
C RSB solution for the teacher-student setting $\quad 46$

C.1 Entropic part of the free-energy 46

C.1.1 Entropic part of the free-energy due to 'bonds' 46

C.1.2 Entropic part of the free-energy due to 'spins' 46

C.2 Interaction part of the free-energy 48

$\begin{array}{lll}\text { C.3 Saddle point equations } & 48\end{array}$

$\begin{array}{lll}\text { C.3.1 Variation of } q_{i}(l) \text { 's } & 48\end{array}$

$\begin{array}{lll}\text { C.3.2 Variation of } G_{i}(l) \text { 's } & 49\end{array}$

$\begin{array}{lll}\text { C.3.3 Variation of } r & 49\end{array}$

C.3.4 Variation of $R \quad 50$

\section{Introduction}

Machine learning by deep neural networks (DNN) is successful in numerous applications [1]. However it remains challenging to understand why DNNs actually work so well. Given the enormous parameter space, which is typically orders of magnitude larger than that of the data space, and the flexibility of non-linear functions used in DNNs, it is not very surprising that they can express complex data [2]. What is surprising is that such extreme machines can be put under control. On one hand, one would naturally fear that learning such huge number of parameters would be extremely time consuming because the fitness landscape is presumably quite complex with many local traps. Moreover over-fitting or poor generalization ability seem unavoidable in such over-parametrized machines. One would not dare to fit a data set of 10 points by a 100 th order polynomial, which does not make sense usually. Quite unexpectedly, these issues seem to be somehow resolved in practice and such extreme machines turned out to be very useful. Thus it is a very interesting scientific problem to uncover what is going on in DNNs [3,4]. This is also important in practice because we wish to use DNNs not merely as mysterious black boxes but control/design them in rational ways.

In this paper we investigate a class of simple machines made of feed-forward networks of layered perceptrons whose depth is $L$ and the width is $N$. Such a machine is parametrized by a configuration of synaptic weights in the hidden layers. For a given pair of inputs/outputs patterns imposed on the input and output layers, there can be different realizations of the synaptic weights which match the constraints. We call each of them as a 'solution'. Following Gardner [5,6], we consider statistical mechanics of the solution space which is compatible with a large number $M=\alpha N$ patterns of training data, in $N \rightarrow \infty$ limit with fixed $\alpha$. For the choice of the training data, we consider two simple scenarios: 1) pairs of purely random inputs/outputs patterns 2) teacher-student setting - pairs of random input and the corresponding output of a teacher machine with random synaptic weights are handed over to a student machine.

From a broader perspective, the setting 1) can be viewed as a random constraint satisfaction problem (CSP) [7,8], which is deeply related to the physics of glass transitions and jamming $[9,10]$. In the context of neural networks it is a standard setting to study the storage capacity [5,11]. If $\alpha$ is small so that the constraint is weak enough, it is natural to expect that the phase space looks like that of a liquid: there are many realizations of machines 
compatible with a given set of constraints and essentially all solutions are continuously connected to each other. Increasing $\alpha$ the system becomes more constrained so that the volume of the solution space shrinks and ultimately vanishes at some critical value $\alpha_{\mathrm{j}}$. This is a SAT/UNSAT (jamming) transition and $\alpha_{\mathrm{j}}$ defines the storage capacity. Before reaching $\alpha_{\mathrm{j}}$, it can happen that solution space becomes clustered into mutually disconnected islands. This is a glass transition and it accompanies some type of replica symmetry breaking (RSB) $[12,13]$. Recently non-trivial glass transitions accompanying continuous replica symmetry breaking, which imply emergence of hierarchical free-energy landscape [13] with ultra-metricity [14], and a common jamming critically as that of the hard-spheres [10] were found in a family of CSPs including a single perceptron problem $[15,16]$ and a family of vectorial spin models [17]. Understanding the nature of such glass transitions and jamming is a fundamental problem in CSPs since it is intimately related to efficiency of algorithms to solve CSPs. In the context of DNN, it is certainly important to understand the characteristics of the free-energy landscape to understand the efficiently of various learning algorithms for DNNs [18-20].

On the other hand the setting 2), is a statistical inference (SI) problem. While the constraint satisfaction problems are related to the physics of glass transitions and jamming, solving a statistical inference problems can be said to be equivalent to searching of a hidden (planted) crystalline state [8]. In the context of neural networks, it is a standard setting to study learning $[6,11]$. As $\alpha$ becomes sufficiently large, the synaptic weights of the student machine start to become closer to those of the teacher machine. If this happens, the student machine starts to generalize: the probability that the student machine yields the same output as the teacher machine with respect to a test data (not used during training), creases with $\alpha$. Although very simple, this setting will provide useful insights on the generalization ability of DNNs.

The key strategy of the present work is to regard a DNN not as a system of long-ranged interaction between the input and output through a highly convoluted non-linear mappings but rather as a system with short-ranged interactions between adjacent layers. This is enabled by the 'internal representation' [21], in which one takes into account not only 'bonds' (synaptic weights) but also 'spins' (neurons) in the hidden layers as dynamical variables which are constrained to satisfy proper inputs/outputs relations at each perceptron embedded in the hidden layers. Representing the states of a neuron associated with $M$-patterns as $M$-component vectorial spins, the system can be represented as a network of elements with large number of components and intermediately dense connections (intermediate between sparse and global coupling) similar to the one studied in [17]. This allows us to investigate the interior of the network through local glass or crystalline order parameters, for both the spins and bonds, which are allowed to vary over the space. We formulate a replica theory to analyze the Gardner volume in terms of these local order parameters using a Gaussian ansatz which allows us to write down the free-energy explicitly as a functional of the space dependent, local order parameters.

The main result of the present paper is that the solutions of the glass/crystalline order parameters of the over-parametrized DNN are quite heterogeneous in space. In both settings 1) and 2), the amplitude of the order parameters close to the inputs/outputs boundaries become finite and take higher values as $\alpha$ increases while the amplitudes decay down to 0 going deeper into the bulk. Moreover, in the case of the setting 1) random inputs/outputs, even the pattern of the replica symmetry breaking (RSB) varies in space : it is most complex close to the boundaries with $k$ (+continuous)-RSB, which becomes $k-1$ (+continuous)-RSB in the next layer, ... down to a replica symmetric $(0$ RSB $)$ state in the central part. The 
thickness $\xi$ of the region around the boundaries where the glass/crystalline order parameters become finite roughly scales as $\xi \propto \ln \alpha$. Thus if the network is deep enough $L>\xi$, the central part of the network remains in the liquid phase: there are too many possibilities left in the central part to meet the constraints imposed at the boundaries. The heterogeneous profile of the order parameters have important implications on how DNNs work.

The organization of the paper is the following. In sec. 2 we introduce the deep neural network model studied in the present paper and explain the two scenarios : 1) random inputs/outputs and 2) teacher-student setting. In sec. 3 we formulate a replica theory to set of a statistical mechanical analysis of the solution space of the deep neural network. In sec. 4 and sec. 5 we study the cases of 1) random inputs/outputs and 2) teacher-student setting respectively using the replica theory. In sec. 6, we present some results of numerical simulations to examine the theoretical predictions. Finally in sec. 7 , we conclude the paper and present some outlook. In the appendices $\mathrm{A}, \mathrm{B}$ and $\mathrm{C}$ we present some details for the theoretical formulation.

\section{Model}

\subsection{Multi-layer feed-forward network}

We consider a simple multi-layer neural network (See Fig. 1) which consists of an input layer $(l=0)$, output layer $(l=L)$ and hidden layers $(l=1,2, \ldots, L-1)$. Each layer consists of $i=1,2, \ldots, N$ neurons $\mathbf{S}_{l, i}$, each of which consists of $M$-component Ising spins $\mathbf{S}_{l, i}=\left(S_{l, i}^{1}, S_{l, i}^{2}, \ldots, S_{l, i}^{M}\right)$ with $S_{l, i}^{\mu}= \pm 1$. Here the label $\mu=1,2, \ldots, M$ is used to distinguish different firing patterns of the neurons (spins). The spins in the inputs/outputs layers represent 'data' provided by external sources. We follow the notation of [17] to represent a factor node, which is a perceptron here, as $\boldsymbol{\square}$. We consider a feed-forward network of $N \mathbf{\square}=N L$ perceptrons A perceptron $\mathbf{\square}$ receives $N$ inputs from the outputs of perceptrons $\mathbf{\square}(k)(k=1,2, \ldots, N)$ in the previous layer, weighted by $\mathbf{J}_{\mathbf{\square}}=\left(J_{\mathbf{\square}}^{1}, J_{\mathbf{\square}}^{2}, \ldots, J_{\mathbf{\square}}^{N}\right)$. Its output S匹 is,

$$
S_{\boldsymbol{\mathbf { a }}}^{\mu}=\operatorname{sgn}\left(\frac{1}{\sqrt{N}} \sum_{k=1}^{N} J_{\mathbf{m}}^{k} S_{\mathbf{a}_{(k)}}^{\mu}\right) \quad \mu=1,2, \ldots, M .
$$

We assume that the synaptic weights $J_{\mathbf{n}}^{k}$ take real numbers normalized such that,

$$
\sum_{k=1}^{N}\left(J^{k}\right)^{2}=N
$$

For simplicity we call the variable for the neurons $\mathbf{S}_{l, i}$ 's simply as 'spins', and the synaptic weights $J^{k}$ s as 'bonds'.

The size of the input data and the number of parameters are

$$
N_{\text {input }}=N M=N^{2} \alpha \quad N_{\text {parameter }}=N_{\mathbf{\varpi}} N=N^{2} L
$$

where we introduced a parameter

$$
\alpha \equiv \frac{M}{N} .
$$




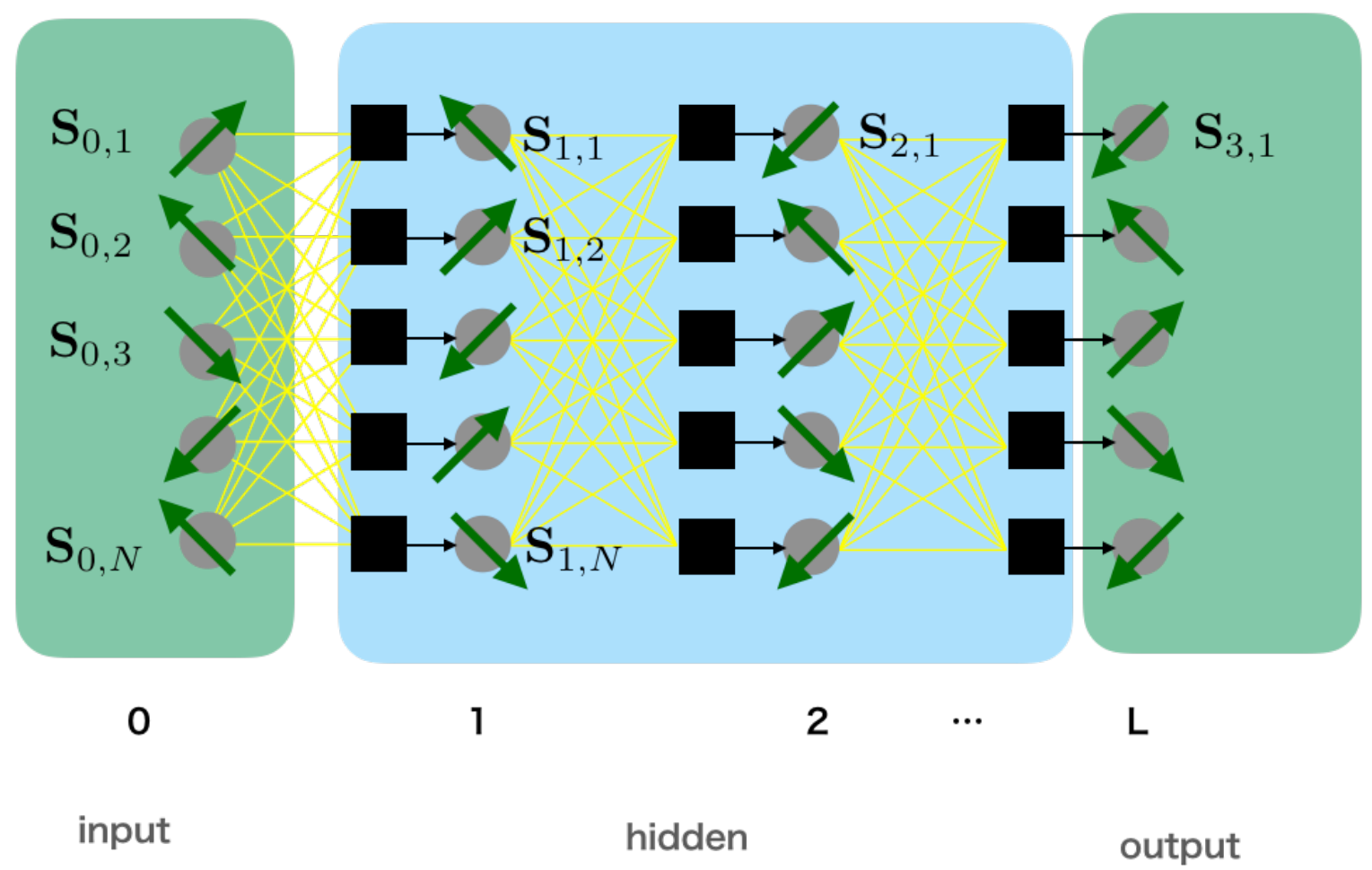

Figure 1: A simple multi-layer perceptron network of depth $L$ and width $N$. In this example the depth is $L=3$. Each arrow represent a $M$-component vector $\operatorname{spin} \mathbf{S}_{i}=\left(S_{i}^{1}, S_{i}^{2}, \ldots, S_{i}^{M}\right)$ with its component $S_{i}^{\mu}= \pm 1$ representing the state of a 'neuron' in the $\mu$-th pattern.

We will consider the limit $N, M \rightarrow \infty$ with fixed $\alpha$. Apparently the system is over parametrized if deep enough $L>\alpha$.

Following the pioneering work by Gardner $[5,6]$ we consider the phase space volume associated with a given set of inputs/outputs patterns represented by $\mathbf{S}_{0}$ and $\mathbf{S}_{L}$, which can be expressed as,

$$
\begin{aligned}
& V\left(\mathbf{S}_{0}, \mathbf{S}_{L}\right)=e^{N M \mathcal{S}\left(\mathbf{S}_{0}, \mathbf{S}_{l}\right)}=\left(\prod_{\mathbf{\square}} \operatorname{Tr}_{\mathbf{J}_{\mathbf{\square}}}\right)\left(\prod_{\square \text { output }} \operatorname{Tr}_{\mathbf{S}_{\mathbf{}}}\right) \prod_{\mu=1}^{M} \prod_{\mathbf{\square}} e^{-\beta V\left(r_{\mathbf{\square}}^{\mu}\right)} \\
& =\prod_{\mathbf{\square}} \prod_{j=1}^{N} \int_{-\infty}^{\infty} d J_{\mathbf{\square}}^{j} \delta\left(\sum_{k=1}^{N}\left(J_{\mathbf{a}}^{k}\right)^{2}-N\right)\left(\prod_{l=1}^{L-1} \prod_{i=1}^{N} \sum_{S_{l, i}^{\mu}= \pm 1}\right) \prod_{\mu=1}^{M} \prod_{\mathbf{\square}} e^{-\beta V\left(r_{\mathbf{m}}^{\mu}\right)}
\end{aligned}
$$

where

$$
e^{-\beta V(r)}=\theta(r)
$$

and we introduced 'gap',

$$
r_{\mathbf{\square}}^{\mu} \equiv S_{\boldsymbol{\square}}^{\mu} \sum_{i=1}^{N} \frac{J_{\mathbf{m}}^{i}}{\sqrt{N}} S_{\mathbf{\square}(i)}^{\mu}
$$


Note that in Eq. (5) summations are took not only over the bonds (synaptic weights) but also over the spins (neurons) in the hidden layers. This is the internal representation [21] by which we can break up the long-ranged interaction between the input and output into short-ranged effective interactions. Actually we can now write the effective Hamiltonian of the system as

$$
\mathcal{H}_{\mathrm{eff}}=\sum_{\mu=1}^{M} \sum_{\mathbf{\square}} V\left(S_{\mathbf{\square}}^{\mu} \sum_{i=1}^{N} \frac{J_{\mathbf{m}}^{i}}{\sqrt{N}} S_{\mathbf{m}(i)}^{\mu}\right)
$$

Let us emphasize that here both the spins $S_{\mathbf{n}_{s}}^{\mu}$ and bonds $J_{\mathbf{n}}^{i}$ are dynamical variables, except for the spins on the boundaries $l=0, L$ which are frozen. From physicist's point of view this is far more convenient than dealing with the very long-ranged interaction. Note however that this trick works because of the simple 'sgn' activation function Eq. (1) we consider in the present paper.

\subsection{Two scenarios for inputs/output patterns}

For the input and output patterns $\mathbf{S}_{0}$ and $\mathbf{S}_{L}$, we consider the following two scenarios.

\subsubsection{Random inputs/outputs}

As the simplest setting, we consider the case of completely random inputs/outputs patterns, which is the standard setting to study the storage capacity of the perceptrons [5]. More precisely all components of $\mathbf{S}_{0, i}=\left(S_{0, i}^{1}, S_{0, i}^{2}, \ldots, S_{0, i}^{M}\right)$ and $\mathbf{S}_{L, i}=\left(S_{L, i}^{1}, S_{L, i}^{2}, \ldots, S_{L, i}^{M}\right)$ for $i=1,2, \ldots, N$ are assumed to be iid random variables which take Ising values \pm 1 . As we noted in the introduction, this setting can be regarded as a random constraint satisfaction problem (CSP).

\subsubsection{Teacher-student setting}

As a complimentary approach, we consider the teacher-student setting, which is a standard setting to study statistical inference problems [8]. We consider two machines : teacher machine and student machine and assume that they have the exactly the same architecture, i. e. the same width $N$ and number of layers $L$.

We assume that the teacher is a 'quenched-random teacher': the set of the synaptic weights $\left\{\left(J_{\mathbf{n}}^{k}\right)_{\text {teacher }}\right\}$ of the teacher machine are iid random variables which obey the normalization Eq. (2). Such a teacher machine is subjected to a set of random inputs $\mathbf{S}_{0, i}=$ $\left(S_{0, i}^{1}, S_{0, i}^{2}, \ldots, S_{0, i}^{M}\right)$ for $(i=1,2, \ldots, N)$ and produces the corresponding set of outputs,

$$
\left(\mathbf{S}_{L, i}\right)_{\text {teacher }}=\left(\left(S_{L, i}^{1}\right)_{\text {teacher }},\left(S_{L, i}^{2}\right)_{\text {teacher }}, \ldots,\left(S_{L, i}^{M}\right)_{\text {teacher }}\right)
$$

The task of the student machine is to try to infer the synaptic weights $\left\{\left(J_{\mathbf{n}}^{k}\right)_{\text {teacher }}\right\}$ of the teacher machine, by construction its own synaptic weights $\left\{\left(J_{\mathbf{n}}^{k}\right)_{\text {student }}\right\}$ such that it successfully reproduces all the outputs of the teacher $\left(\mathbf{S}_{L, i}\right)_{\text {teacher }}$ starting from the same input data.

Note that the student is given the full information of the input $\mathbf{S}_{0, i}$ and the output of the teacher $\left(\mathbf{S}_{L, i}\right)_{\text {teacher }}$ plus full information of the architecture of the teacher. In the context of statistical inference, this is an idealized situation called as Bayes optimal case [8] and we limit ourselves to this in the present paper for simplicity. 


\subsection{Modified model}

Unfortunately our original problem Eq. (5) cannot be solved exactly so that we will make a kind of Gaussian ansatz as we explain later. We will also show that this is equivalent to replace the gap function of the original model Eq. (7) by a modified one,

$$
r_{\boldsymbol{\square}}^{\mu} \equiv \sum_{i=1}^{N} \frac{J_{\mathbf{\bullet}}^{i}}{\sqrt{N}}\left(\sum_{\nu=1}^{M} \frac{\xi^{\mu \nu}}{\sqrt{M}} S^{\nu} S_{\mathbf{\square}}^{\nu}(i)\right)
$$

where $\xi^{\mu \nu}$ are quenched random Gaussian variables with zero mean and variance one. Note that the original model corresponds to $\xi^{\mu \nu}=\sqrt{M} \delta_{\mu \nu}$. Clearly the constraints have become looser in the modified model.

\section{Replica theory}

Now let us formulate a replica approach to study the solution space of the deep neural network. To study the case of random inputs/outputs (sec. 2.2.1) we consider $n$ replicas $a=1,2, \ldots, n$ which are independent machines subjected to the common set of inputs $\mathbf{S}_{0, i}$ and outputs $\mathbf{S}_{L, i}$ for $i=1,2, \ldots, N$. For the case of the teacher-student setting (sec. 2.2.2) we consider $n=1+s$ replicas, with replica $a=0$ to represent the teacher machine and other replicas $a=1,2, \ldots, s$ to represent the student.

\subsection{Order parameters}

Given the dense connections of the network, we naturally introduce 'local' order parameters (see [17]),

$$
Q_{a b, \boldsymbol{\square}}=\frac{1}{N} \sum_{i=1}^{N}\left(J_{\mathbf{\square}}^{i}\right)^{a}\left(J_{\mathbf{\square}}^{i}\right)^{b} \quad q_{a b, \boldsymbol{\varpi}}=\frac{1}{M} \sum_{\mu=1}^{M}\left(S_{\mathbf{\square}}^{\mu}\right)^{a}\left(S_{\mathbf{\square}}^{\mu}\right)^{b}
$$

Note that the normalization condition for the bonds Eq. (2) and the spins (which take Ising values \pm 1 ) implies $Q_{a a}, \mathbf{\square}=q_{a a}, \mathbf{\square}=1$.

There are two comments regarding some trivial symmetries left in the system. First, the system is obviously symmetric under permutations of the labels put on the data $\mu=$ $1,2, \ldots, M$. The labels put on different replicas could be permuted differently. In Eq. (11) it is assumed that all replicas follow the same labels breaking the permutation symmetry. Second, the system is symmetric under permutations of perceptrons within the same layer and the permutations could be done differently on different replicas. In Eq. (11) this permutation symmetry is also broken. 


\subsection{Replicated Gardner volume}

The replicated phase space volume (the Gardner volume) can be written as,

$$
\begin{aligned}
& V^{n}\left(\mathbf{S}_{0}, \mathbf{S}_{L}\right)=e^{N M \mathcal{S}_{n}\left(\mathbf{S}_{0}, \mathbf{S}_{l}\right)}
\end{aligned}
$$

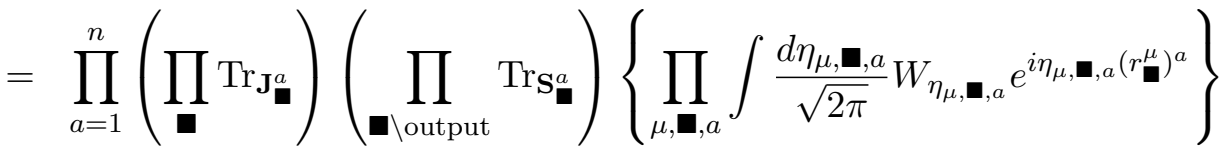

$$
\begin{aligned}
& =\left(\prod_{\mu, \mathbf{\square}, a} \int \frac{d \eta_{\mu, \mathbf{\square}, a}}{\sqrt{2 \pi}} W_{\eta_{\mu, \mathbf{m}, a}}\right)\left(\prod_{\mathbf{\square}} \operatorname{Tr}_{\mathbf{J}} \mathbf{\square}_{\mathbf{\square}}\right)\left(\prod_{\mathbf{\square} \backslash \text { output }} \operatorname{Tr}_{\mathbf{S}_{\mathbf{\bullet}}^{a}}\right)
\end{aligned}
$$

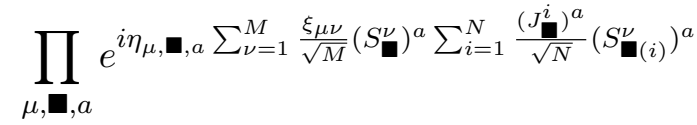

where we introduced a Fourier representation,

$$
e^{-\beta V(r)}=\int \frac{d \eta}{\sqrt{2 \pi}} W_{\eta} e^{-i \eta r}
$$

As shown in appendix A, following similar steps as in [17] we can obtain the replicated free-entropy functional in terms of the order parameters Eq. (11) in the limit $N, M \rightarrow \infty$ with fixed $\alpha=M / N$. As explained in appendix A.2 the free-energy functional is an approximate one (Gaussian approximation for the fluctuation of spins) for the original model but exact for the modified model introduced in sec. 2.3.

Given the structure of the network it is natural to assume that order parameters are the same within each layer layer $l=0,1,2, \ldots, L$,

$$
Q_{a b, \boldsymbol{\varpi}}=Q_{a b}(l) \quad q_{a b, \boldsymbol{\varpi}}=q_{a b}(l),
$$

Then the final expression is given by,

$$
\begin{aligned}
S_{n}[\{\hat{Q}(l), \hat{q}(l)\}] & =\frac{1}{\alpha} \sum_{l=1}^{L} S_{\text {ent,bond }}[\hat{Q}(l)]+\sum_{l=1}^{L-1} S_{\text {ent,spin }}[\hat{q}(l)] \\
& -\sum_{l^{\prime}=1}^{L} \mathcal{F}_{\text {int }}\left[\hat{q}\left(l^{\prime}-1\right), \hat{Q}\left(l^{\prime}\right), \hat{q}\left(l^{\prime}\right)\right]
\end{aligned}
$$

where the entropic part of the free-energy due to the 'bonds' $S_{\text {ent,bond }}[\hat{Q}]$ reads as,

$$
S_{\text {ent,bond }}[\hat{Q}]=\frac{1}{2} \ln \operatorname{det} \hat{Q}
$$

and that due to the 'spins' $S_{\text {ent }}[\hat{q}]$ reads as,

$$
S_{\text {ent,spin }}[\hat{q}]=\sum_{a, b} \frac{1}{2} \varepsilon_{a b}^{*} q_{a b}+\left.\ln e^{-\frac{1}{2} \sum_{a, b} \varepsilon_{a b}^{*} \frac{\partial^{2}}{\partial h_{a} \partial h_{b}}} \prod_{a} 2 \cosh \left(h_{a}\right)\right|_{\left\{h_{a}=0\right\}} .
$$

with $\varepsilon_{a b}^{*}=\varepsilon_{a b}^{*}[\hat{q}]$ determined through,

$$
q_{a b}=-\left.\left.\frac{\delta}{\delta \varepsilon_{a b}} \ln e^{-\sum_{a<b} \varepsilon_{a b} \frac{\partial^{2}}{\partial h_{a} \partial h_{b}}} \prod_{a} 2 \cosh \left(h_{a}\right)\right|_{\left\{h_{a}=0\right\}}\right|_{\varepsilon_{a b}=\varepsilon_{a b}^{*}[\hat{q}]}
$$


Finally the interaction part of the free-energy reads as,

$$
-\mathcal{F}_{\text {int }}[\hat{q}(l-1), \hat{Q}(l), \hat{q}(l)]=\left.\ln e^{\frac{1}{2} \sum_{a b} q_{a b}(l-1) Q_{a b}(l) q_{a b}(l) \frac{\partial^{2}}{\partial_{h_{a}} \partial_{h_{b}}}} \prod_{a=1}^{n} e^{-\beta V\left(h_{a}\right)}\right|_{h_{a}=0}
$$

The order parameters must verify saddle point equations,

$$
\begin{array}{rlrl}
\left.\frac{\partial}{\partial Q_{a b}(l)} \partial_{n} S_{n}[\{\hat{Q}(l), \hat{q}(l)\}]\right|_{n=0}=0 & l=1,2, \ldots, L \\
\left.\frac{\partial}{\partial q_{a b}(l)} \partial_{n} S_{n}[\{\hat{Q}(l), \hat{q}(l)\}]\right|_{n=0}=0 & l=1,2, \ldots, L-1
\end{array}
$$

The above general formulation can be adapted for the two scenarios introduced in sec. 2.2 as follows,

- Random inputs/outputs

In the case of random inputs/outputs (sec. 2.2.1) we consider the free-energy functional,

$$
\frac{-\beta F[\{\hat{Q}(l), \hat{q}(l)\}]}{N M}=\frac{\partial_{n} \overline{V^{n}\left(\mathbf{S}_{0}, \mathbf{S}_{L}\right)} \mathbf{S}_{0},\left.\mathbf{S}_{L}\right|_{n=0}}{N M}=\left.\partial_{n} S_{n}[\{\hat{Q}(l), \hat{q}(l)\}]\right|_{n=0} .
$$

The presence of the imposed random inputs/outputs can be specified by providing values of $q_{a b}(0)$ and $q_{a b}(L)$. Since both the teacher and student machines are subjected to the same inputs and outputs, we can simply set,

$$
q_{a b}(0)=q_{a b}(L)=1 .
$$

As we discuss later we will also consider the case of fluctuating boundary condition.

- Teacher-student setting

In the case of the teacher-student setting (sec. 2.2.2) we consider instead a the so called Franz-Parisi potential [22],

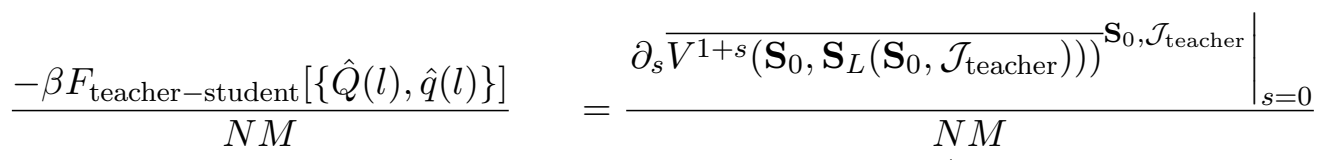

$$
\begin{aligned}
& =\left.\partial_{s} S_{1+s}[\{\hat{Q}(l), \hat{q}(l)\}]\right|_{s=0} .
\end{aligned}
$$

where the over-line denotes the average over the imposed random inputs imposed commonly on both the teacher and student machines. The outputs are just those of the teacher machine $a=0, \mathbf{S}_{L}\left(\mathbf{S}_{0}, \mathcal{J}_{\text {teacher }}\right)$ which are of course functions of the inputs $\mathbf{S}_{0}$ and the synaptic weights of the teacher machine $\mathcal{J}_{\text {teacher }}=\left\{\left(J_{\mathbf{n}}^{k}\right)_{\text {teacher }}\right\}$. Since both the teacher and student machines are subjected to the same inputs, we set,

$$
q_{a b}(0)=1
$$

In addition, since the outputs of the student machine are forced to agree perfectly with that of the teacher machine we set,

$$
q_{a b}(L)=1
$$




\subsection{Parisi's ansatz}

\subsubsection{Random inputs/outputs}

In the case of random inputs/outputs (sec. 2.2.1) we have $n$ replicas $a=1,2, \ldots, n$. Then it is natural to consider the standard Parisi's ansatz with $k$-step RSB (including RS as $k=0$ and continuous RSB as $k=\infty$ ) [12,13] (See Fig. 2),

$$
\begin{array}{rlr}
Q_{a b}(l)=\sum_{i=0}^{k+1} Q_{i}(l)\left(I_{a b}^{m_{i}}-I_{a b}^{m_{i+1}}\right) & l=1,2, \ldots, L \\
q_{a b}(l)=\sum_{i=0}^{k+1} q_{i}(l)\left(I_{a b}^{m_{i}}-I_{a b}^{m_{i+1}}\right) & l=1,2, \ldots, L-1 \\
\varepsilon_{a b}(l)=\sum_{i=0}^{k} \varepsilon_{i}(l)\left(I_{a b}^{m_{i}}-I_{a b}^{m_{i+1}}\right) & l=1,2, \ldots, L-1
\end{array}
$$

where $q_{k+1}(l)=Q_{k+1}(l)=1$ and $I_{a b}^{m}$ being a generalized ('fat') Identity matrix of size $n \times n$ composed of blocks of size $m \times m$. In the Parisi's ansatz one considers

$$
1=m_{k+1}<m_{k}<\ldots<m_{1}<m_{0}=n
$$

which becomes

$$
0=m_{0}<m_{1}<\ldots<m_{k}<m_{k+1}=1
$$

in the $n \rightarrow 0$ limit. In the $k \rightarrow \infty$ limit, the matrix elements can be parametrized by functions $q(x, l), Q(x, l)$ and $\epsilon(x, l)$ defined in the range $0 \leq x \leq 1$ (See Fig. $2 \mathrm{~d}$ )).

The order parameter functions encode characteristics of the complex free-energy landscape [13]. For example the distribution functions of the overlaps between two replicas (two independent machines) can be related to the order parameter functions as,

$$
\begin{array}{cc}
P(q, l)=\frac{d x(q, l)}{d q} & x(q, l)=\int_{0}^{q} d q^{\prime} P\left(q^{\prime}, l\right) \\
P(Q, l)=\frac{d x(Q, l)}{d Q} & x(Q, l)=\int_{0}^{Q} d Q^{\prime} P\left(Q^{\prime}, l\right)
\end{array}
$$

Thus $x(q, l)(x(Q, l))$ is the probability that the mutual overlap of the spin (bond) patterns at $l$-th layer between two machine are smaller than $q(Q)$. Equivalently $1-x(q, l)(1-x(Q, l))$ is the probability that the mutual overlap of the spin (bond) patterns at $l$-th layer between two machine are larger than $q(Q)$.

The functions $q(x, l)$ and $Q(x, l)$ are expected to have a 'plateau' close to $x=1$, which gives rise to a delta functions in the overlap distribution functions $P(q, l)$ and $P(Q, l)$. As usual we regard the plateau values as the self-overlaps of the meta-stable states or the EdwardsAnderson order parameters $q_{\mathrm{EA}}(l)$ and $Q_{\mathrm{EA}}(l)$. In practice we will use the values of $q_{k}$ and $Q_{k}$ in the $k$-RSB ansatz as the Edwards-Anderson order parameters.

Analysis of the free-energy functional $-\beta F[\{\hat{Q}(l), \hat{q}(l)\}] / M N$ Eq. (22) can be done using these matrices in Eq. (15)-Eq. (19). In appendix B, we present details of the RSB solution. 
a)

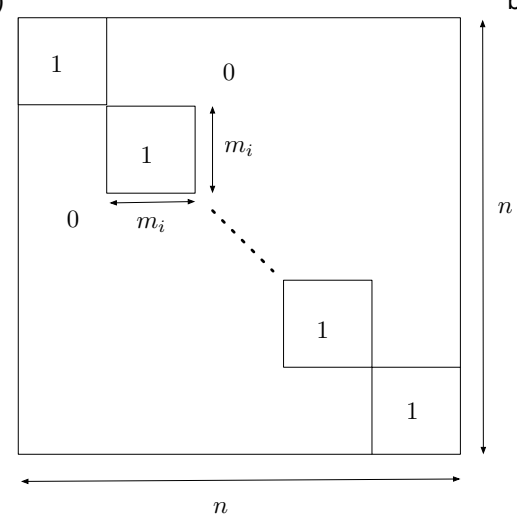

b)

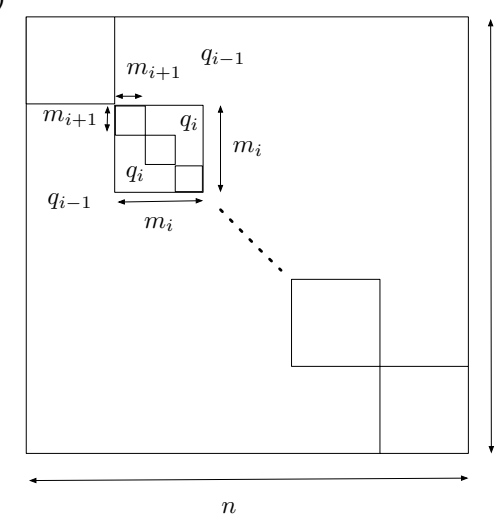

c)

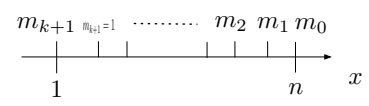

d)

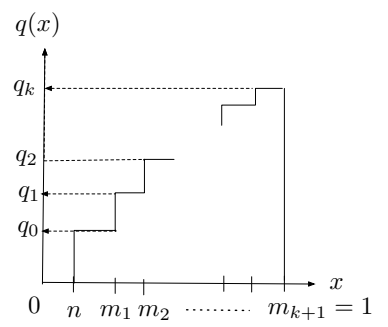

Figure 2: Parametrization of the Parisi's matrix a) the 'fat' identity matrix $I_{a b}^{m_{i}}$ b) Parisi's matrix given by Eq. (28) (Eq. (27) and Eq. (29) have the same structure with $q_{i}$ 's replaced by $Q_{i}$ 's and $\epsilon_{i}$ 's.) c) the hierarchy of the sizes $m_{i}$ of the sub-matrices d) the $q(x)$ function with $0<n<1(Q(x), \epsilon(x)$ functions have the same structure).

\subsubsection{Teacher-student setting}

For the teacher-student setting (sec. 2.2.2) we have to modify the matrices $\hat{Q}, \hat{q}$ and $\hat{\epsilon}$ slightly to include $a=0$ for the teacher machine in addition to $a=1,2, \ldots, s$ for the student as shown in Fig. 3. We denote the modified matrices as $\hat{Q}^{1+s}, \hat{q}^{1+s}$ and $\hat{\epsilon}^{1+s}$. The sub-matrices $\hat{q}^{s}, \hat{Q}^{s}$ and $\hat{\epsilon}^{s}$ are for the student for which we assume the same hierarchical structure as before Eq. (27)-Eq. (29).

Analysis of the Franz-Parisi potential $-\beta F_{\text {teacher-student }}[\{\hat{Q}(l), \hat{q}(l)\}] / M N$ Eq. (24) can be done using these matrices in Eq. (15)-Eq. (19). In appendix C, we present details of the RSB solution.

a)

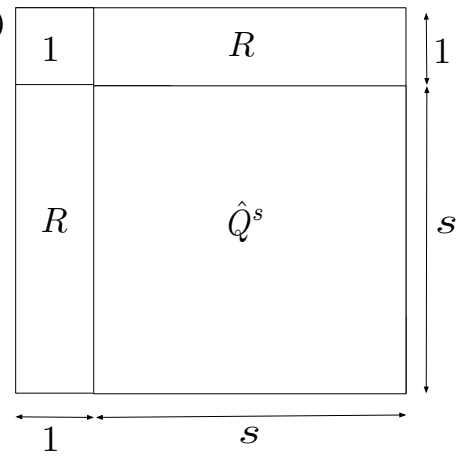

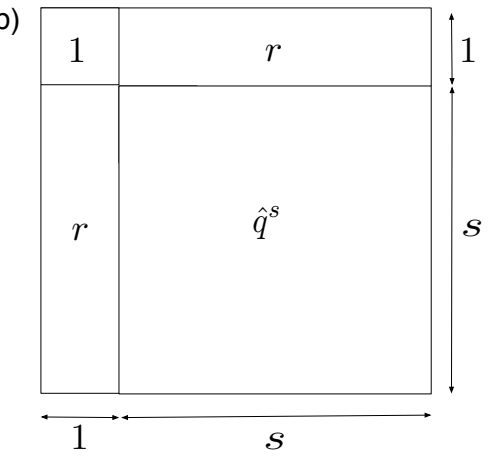

Figure 3: Parametrization of the Parisi's matrices for the teacher-student setting : a) $\hat{Q}^{1+s}$ b) $\hat{q}^{1+s}$ and c) $\hat{\epsilon}^{1+s}$. For the sub-matrices $\hat{q}^{s}, \hat{Q}^{s}$ and $\hat{\epsilon}^{s}$ of size $s \times s$ we assume the same hierarchical structure as those in Eq. (27)-Eq. (29) (see Fig. 2) but with $n$ replaced by $s$. 


\section{Random inputs/outputs}

Here we analyze in detail the case of random inputs/outputs introduced in sec. 2.2.1 by the replica theory using the ansatz explained in sec. 3.3.1. We will consider not only the usual case of 'quenched' random inputs/outputs [5] but also the case of 'fluctuating' random inputs/outputs.

\subsection{Quenched boundary}

Let us start with the case of the usual quenched boundary : the configurations of the spins on the input and output boundaries are chosen randomly and then fixed (Fig. 4). It amounts to choose 'replica symmetric' boundaries, because all replicas are subjected to the same inputs/outputs pattern,

$$
q_{a b}(0)=1 \quad q_{a b}(L)=1
$$

or

$$
\begin{aligned}
& q_{0}(0)=q_{0}(L)=1 \\
& q_{i}(0)=q_{i}(L)=0 \quad(i=1,2, \ldots, k)
\end{aligned}
$$

in terms of the Parisi's matrix Eq. (28).

We analyze the saddle point solutions numerically as described in sec. B.3.3. In the following we present results using $k=100$ step RSB and the depth of the system $L=5-20$. Because of the choice of the boundary condition, the system becomes symmetric with respect to reflections at the center: we confirmed that the solutions satisfy $q_{i}(l)=q_{i}(L-l)$ and $Q_{i}(l)=Q_{i}(L-l)$. However it must be noted that this is an artifact of our theory because the original system (See Fig. 1) do not have this symmetry. The origin can be traced back to the Gaussian approximation made in the derivation of the free-energy functional in terms of the order parameters.

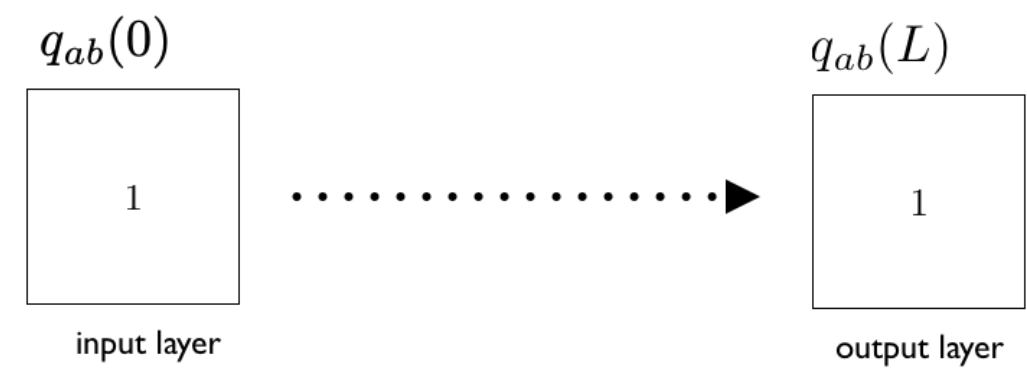

Figure 4: Quenched boundary

\subsubsection{Liquid phase}

For small $\alpha=M / N$ we find the whole system is in the liquid phase where the glass order parameters are all zero: for $i=1,2, \ldots, k q_{i}(l)=0(l=1,2, \ldots, L-1)$ and $Q_{i}(l)=0$ $(l=1,2, \ldots, L)$. This means that the parameter space is so large compared with the number 
of imposed constraints so that there are simply too many solutions compatible with the constraints.

\subsubsection{1st glass transition}
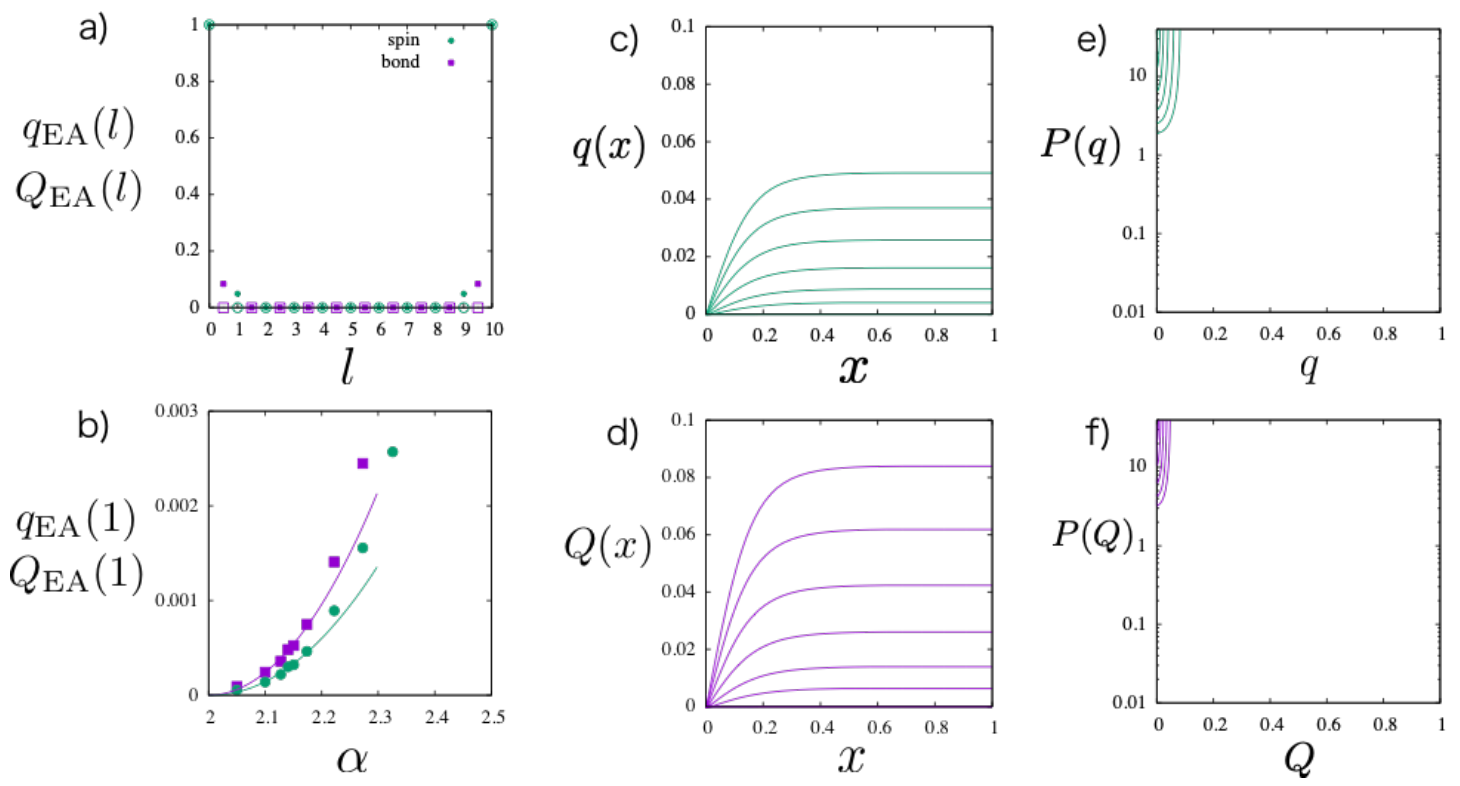

Figure 5: Glass order parameters close to the 1st glass transition: a) spatial profile of the Edwards-Anderson (EA) order parameter for spins $q_{\mathrm{EA}}(l)\left(=q_{k=100}(l)\right)$ and bonds $Q_{\mathrm{EA}}(l)\left(=Q_{k=100}(l)\right.$ slightly before $\alpha=2.0$ (empty symbols)/after $\alpha=3.125$ (filled symbols) the 1st glass transition. The depth is $L=10$ in this example. b) Evolution of the EA order parameters $q_{\mathrm{EA}}(1)=q_{k}(1)$ and $Q_{\mathrm{EA}}(1)=Q_{k}(1)$ at the 1st layer after passing the critical point of the 1 st glass transition $\alpha_{\mathrm{g}}(1) \simeq 2.03$. c),d) Glass order parameter function $q(x, l)$ for spins and $Q(x, l)$ for bonds at the 1st layer $l=1$ at around the 1st glasstransition. Here $1 / \alpha=0.32,0.34,0.36,0.38,0.40,0.42$ from the top to the bottom. e),f) the overlap distribution function of spins $P(q)=d x(q) / d q$ and bonds $P(Q)=d x(Q) / d Q$ (see Eq. (33)).

With increasing $\alpha$, the system becomes more constrained. We find a continuous glass transition at $\alpha_{\mathrm{g}}(1) \simeq 2.03$ on the two layers $l=1, L-1$ just beside the quenched inputs/outputs boundaries as shown in Fig. 5 a). The rest of the system $(l=2,3, \ldots, L-2)$ remains in the liquid phase $q_{\mathrm{EA}}(l)=Q_{\mathrm{EA}}(l)=0$ at this stage. As shown in Fig. $\left.5 \mathrm{~b}\right)$ the Edwards-Anderson (EA) order parameters of the spins $q_{\mathrm{EA}}(l)=q_{k}(l)$ and bonds $Q_{\mathrm{EA}}(l)=Q_{k}(l)$ at the 1st layer $l=1$ grow continuously across the critical point $\alpha_{\mathrm{g}}(1)$. Exactly the same happens at the last layer $l=L-1$.

As shown in Fig. 5 c),d), the functions $q(x, l)$ an $Q(x, l)$ at the 1st layer $l=1$ (and the same for $l=L-1$ ) are continuous functions of $x$ with plateaus at $q_{\mathrm{EA}}$ and $Q_{\mathrm{EA}}$ for some range $x_{1}(\alpha)<x<1$ with $x_{1}(\alpha)$ decreasing with $\alpha$. Thus the replica symmetry is fully broken much as in the SK model for spinglasses $[12,13]$. Correspondingly the overlap distribution functions Eq. (33) $P(q)=d x(q) / d q$ and $P(Q)=d x(Q) / d Q$ shown in Fig. 5 e),f), exhibit 
delta peaks at $q=q_{\mathrm{EA}}, Q=Q_{\mathrm{EA}}$ plus non-trivial continuous parts extending down to $q=0$ and $Q=0$.

\subsubsection{2nd glass transition}
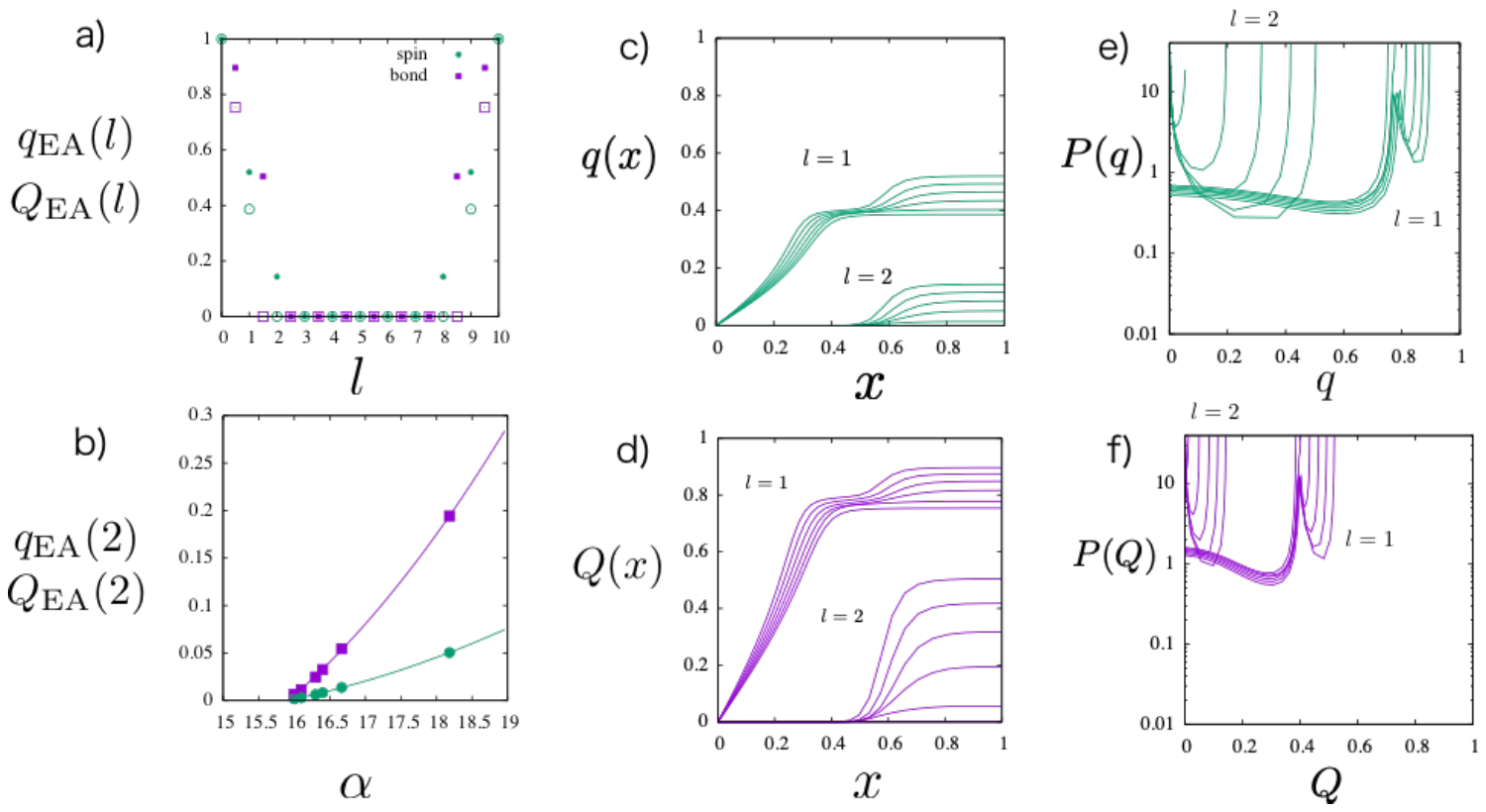

Figure 6: Glass order parameters close to the 2nd glass transition: a) spatial profile of the Edwards-Anderson (EA) order parameter for spins $q_{\mathrm{EA}}(l)$ and bonds $Q_{\mathrm{EA}}(l)$ slightly before $\alpha=15.38$ (empty symbols)/after $\alpha=25$ (filled symbols) the 2 nd glass transition. The depth is $L=10$ in this example. b) Evolution of the EA order parameters $q_{\mathrm{EA}}(2)$ and $Q_{\mathrm{EA}}(2)$ at the 2nd layer after passing the critical point of the 2nd glass transition $\alpha_{\mathrm{g}}(2) \simeq 15.9$. c),d) Glass order parameter function $q(x, l)$ for spins and $Q(x, l)$ for bonds at the 1st layer $l=2$ at around the 2nd glasstransition. Here $1 / \alpha=0.04,0.045,0.05,0.055,0.06,0.065$ from the top to the bottom. e),f) the overlap distribution function of spins $P(q)=d x(q) / d q$ and bonds $P(Q)=d x(Q) / d Q$ Eq. (33).

Increasing $\alpha$ further we find 2 nd glass transition at $\alpha_{\mathrm{g}}(2) \simeq 15.9$ by which two layers $l=2, L-2$ become included in the glass phase while the rest of the system $l=3,4, \ldots, L-3$ still remains in the liquid phase as shown in Fig. 6 a). The glass phase has grown a step further into the interior. The transition is again a continuous one as can be seen in Fig. 6 b) where we display the EA order parameters $q_{\mathrm{EA}}(l)=q_{k}(l)$ and $Q_{\mathrm{EA}}(l)=Q_{k}(l)$ at $l=2$. Exactly the same happens on the other side at $l=L-2$.

As shown in Fig. $6 \mathrm{c}$ ),d), the functions $q(x, l)$ an $Q(x, l)$ at the 2 nd layer $l=2$ (and the same for $l=L-2)$ are continuous functions of $x$ with plateaus at $q_{\mathrm{EA}}(2)$ and $Q_{\mathrm{EA}}(2)$ in some range $x_{2}(\alpha)<x<1$ with $x_{2}(\alpha)$ decreasing with $\alpha$. A marked difference with respect to the 1st glass transition which happened at $l=1$ (and $l=L-1$ ) is that the order parameters becomes finite only in some range $x_{2}(\alpha) \lesssim x<1$. As the result it looks approximately like a step function with the step located at $x_{2}(\alpha)$. As shown in Fig. 6 e),f), this amount to induce 
a delta peak not only at $q_{\mathrm{EA}}(2)\left(Q_{\mathrm{EA}}(2)\right)$ but also at $q=Q=0$ in the distribution of the overlaps.

Remarkably, the 2nd glass transition induce another continuous glass transition on the 1st layer $l=1$ (and $l=L-1$ ) which were already in a glass state. As can be seen in Fig. 6 c),d), an internal step like structure emerge continuously within the region where the glass order parameter was flat $x_{1}(\alpha)<x<1$ before the 2 nd glass transition. As shown in Fig. 6 e), f), the emergence of the internal step amount to a continuous splitting of the delta peak at $q_{\mathrm{EA}}(1)$ $\left(Q_{\mathrm{EA}}(1)\right)$ into two peaks (plus a continuous part in between) meaning that the glass phase has become more complex. This means the meta-stable glassy states have been split into multiple glassy states. In a sense this is similar to the Gardner transition found originally in Ising $p$-spin spin-glass models [23] and in the hard-sphere glass in large-dimensional limit [10,24].

Let us note that the internal step of the glass order parameters on the 1st layer $l=1$ (and $l=L-1)$ is located around $x_{2}(\alpha)$ being synchronized with the step on the 1st layer $l=1$ (and $l=L-1$ ). This 'matching' of the glass order parameters between the different layers have interesting implications as we discuss later.

\subsubsection{More glass transitions}
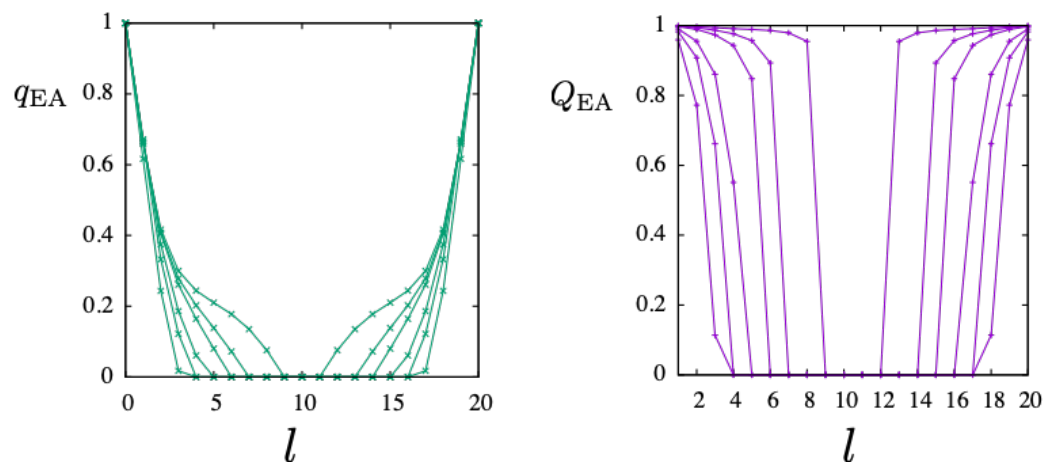

Figure 7: The spatial profile of the EA order parameters $q_{\mathrm{EA}}(l)=q_{k}(l)$ and $Q_{\mathrm{EA}}(l)=Q_{k}(l)$ at $1 / \alpha=0.02,0.01,0.005,0.001,0.0005,0.00025$. Here $L=20$.

Now it is easy to imagine that glass phase will grow further invading the liquid phase by increasing $\alpha$. As we show in Fig. 7, this is indeed the case. We observe that the glass transition point $\alpha_{\mathrm{g}}(l)$ of the $l$-th layer (and $L-l$ ) grows exponentially with $l$. In other words, the 'penetration depth' of the glass phase $\xi_{\text {glass }}$ grows roughly as,

$$
\xi_{\text {glass }} \sim \ln \alpha
$$

As $\alpha$, i. .e. the number of constraints increase, the allowed phase space become suppressed. In Fig. 8 we display $x(Q, l)=\int_{0}^{Q} d Q P(Q, l)$ Eq. (33). The latter is the probability that two replicas (two machines learning independently) subjected to the same inputs/outputs have mutual overlap of the bonds at $l$-th layer smaller than $Q$. As can be seen in the figure, the probability appear to decay as $1 / \sqrt{\alpha}$ for all $l$ and $Q$. This implies two independent machines become more and more similar as the number of constraints increases. 


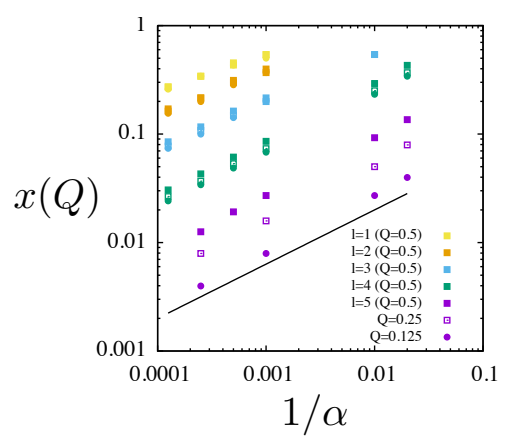

Figure 8: Decay of $x(Q)$ with increasing $\alpha$. Here values of $x(Q, l)$, which is the inverse function of $Q(x, l)$, are shown at various $Q$ and layers $l$. The slope of the straight line is $1 / 2$.

As shown in Fig. 9, the glass order parameter functions become quite complex at large values of $\alpha$. Closer to the boundaries, the system has experienced larger numbers of successive glass transitions which leave behind river terrace like structure with many steps in the glass order parameter functions. This means distribution functions of the overlap with many delta peaks. The steps of the glass order parameter functions at different layers appear to be aligned to each other.

Let us discuss implications of the characteristic glass order parameters $q(x, l)$ and $Q(x, l)$ shown in Fig. 5, 6 and 9. The essence of the river terrace like glass order parameter functions can be sketched schematically as shown in Fig. 10 a). Here we have simplified the picture representing the functions by staircases neglecting their rounding. Comparing the river terraces at different layers we notice an interesting feature that the steps at different layers are synchronized: they are all located at the same positions, $\ldots, x_{l-1}, x_{l}, x_{l+1}, \ldots$. The river terraces reflect successive glass transitions in the following way. At the $n$-th glass transition, finite glass order parameter emerge continuously in the interval $x_{n}(\alpha)<x<1$ at the $n$-th (and $(L-n)$-th) layer. The glass order parameter functions at layers between the $n$-th layer and the boundary, those at $l=1,2, \ldots, n-1$ (and the corresponding layers on the other side), which are already in the glass phase, acquire additional steps in the same interval $x_{n}(\alpha)<x<1$. For a given $\alpha$, the layers included in the glass phase is $l=1,2, \ldots, n$ (and the corresponding ones on the other side) where $n$ is such that $\alpha_{\mathrm{g}}(n)<\alpha<\alpha_{\mathrm{g}}(n+1)$. Due to the successive glass transitions $1,2, \ldots, n$, the $l$-th (and $L-l$ th) layer with $1 \leq l \leq n$ have steps at $0<x_{l}(\alpha)<x_{l+1}(\alpha) \ldots<x_{n}(\alpha)<1$. Correspondingly the overlap distribution functions $P(Q, l)$ and $P(q, l)$ exhibit delta peaks at $q\left(x_{l}, l\right)<q\left(x_{l+1}, l\right) \ldots<q\left(x_{n}, l\right)$ plus another delta peak at $q=0$ for $2 \leq l \leq n$.

At each layer, we have a non-trivial glass order parameter functions $q(x, l)$ as shown in Fig. 10 a), which means hierarchical clustering of replicas as shown schematically in Fig. 10 b) and then it implies the hierarchical free-energy landscape with basins, meta-basins,... as shown schematically in Fig. 10 c) [13]. Here let us explain the correspondences. In panel b), we show clusters of replicas encoded in the glass order parameter functions shown in panel a). Clusters of the same color represent those associated with the same step in the glass order parameter function at some $x$. The size of a cluster associated with a $x$ at a given layer $l$ represents the strength of fluctuation $1-q(x, l)$,i. e. typical distance between the replicas belonging to the cluster, which increases with decreasing $x$ and/or going away from 
a)

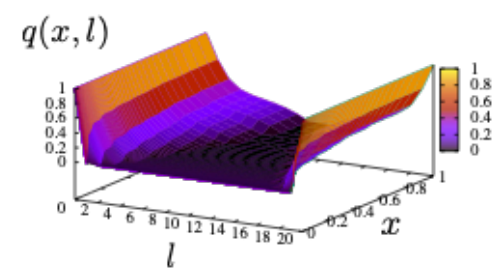

b)

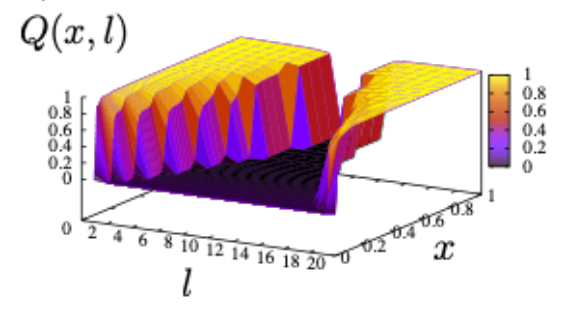

c)

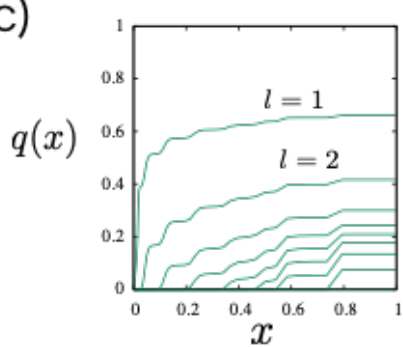

d)

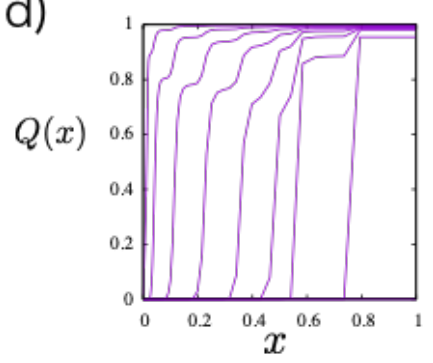

e)

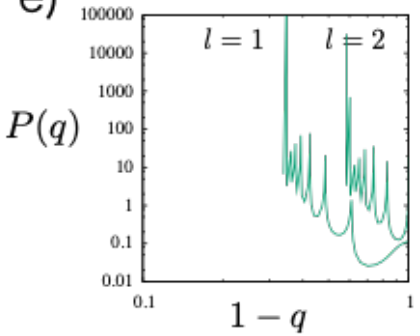

f)

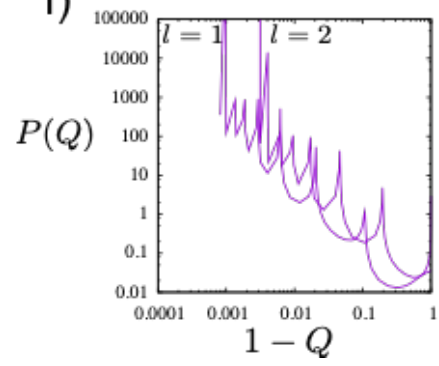

Figure 9: Glass order parameter functions under stronger constraints $\alpha=4000$. In this example $L=20$ and the central layers at $l=8,9$ still remain in the liquid phase. a),b) 3 dimensional plots of $q(x, l)$ and $Q(x, l) . \mathrm{c}), \mathrm{d})$ the same in 2 dimensional plots. e,f) the corresponding overlap distribution functions Eq. (33) at $l=1,2$ (for clarity others at $l=$ $3,4, \ldots$ are not shown).

the boundary $l=1,2, \ldots$. The river terrace of $q(x, l)$ with multiple steps at different $x$ means hierarchical organization of clusters at each layer $l$ : clusters of replicas which are close to each other are enclosed in a meta-cluster of replicas at lower $x$ in which replicas can be a bit more separated from each other, and so on.

To understand meaning of the hierarchical clustering of replicas along the $x$-axis, it is useful to consider two independent machines subjected to the same inputs/outputs which can be regarded as two replicas. Recalling that $x$ has a probabilistic meaning as given in Eq. (33), we notice that the two machines at a given layer $l$ belong to the same cluster at level $x$ with the probability $1-x$ which is larger for smaller $x$. Therefore, even if the two machines do not belong to the same cluster at a given level $x$ with some overlap $q$, they may still belong to a common 'meta' cluster at some lower $x$ with lower overlap $q$. The hierarchical organization of clusters imply the hierarchical free-energy landscape as shown in panel c): small valleys are group into meta-valleys. The free-energies of the valleys within the same meta-valley are correlated [13]. The strength of fluctuation $1-q(x, l)$ at different level $x$ can be understood as effective 'flatness' of the free-energy landscape at corresponding levels.

The most important feature of the result discussed in this section is that the free-energy landscape evolves in space in such a way that it progressively become less complex and flatter as we go deeper into the interior. For a given $\alpha$, the penetration $\operatorname{depth} \xi_{\text {glass }}(\alpha) \sim \ln \alpha$ is finite. So that in deep enough network $L / 2>\xi_{\text {glass }}(\alpha)$, the interior remains in the liquid phase. Moreover the fact that the river terraces of the glass order parameter functions at different layers are synchronized to each other with common positions of the steps at $x_{1}<x_{2} \ldots$, 


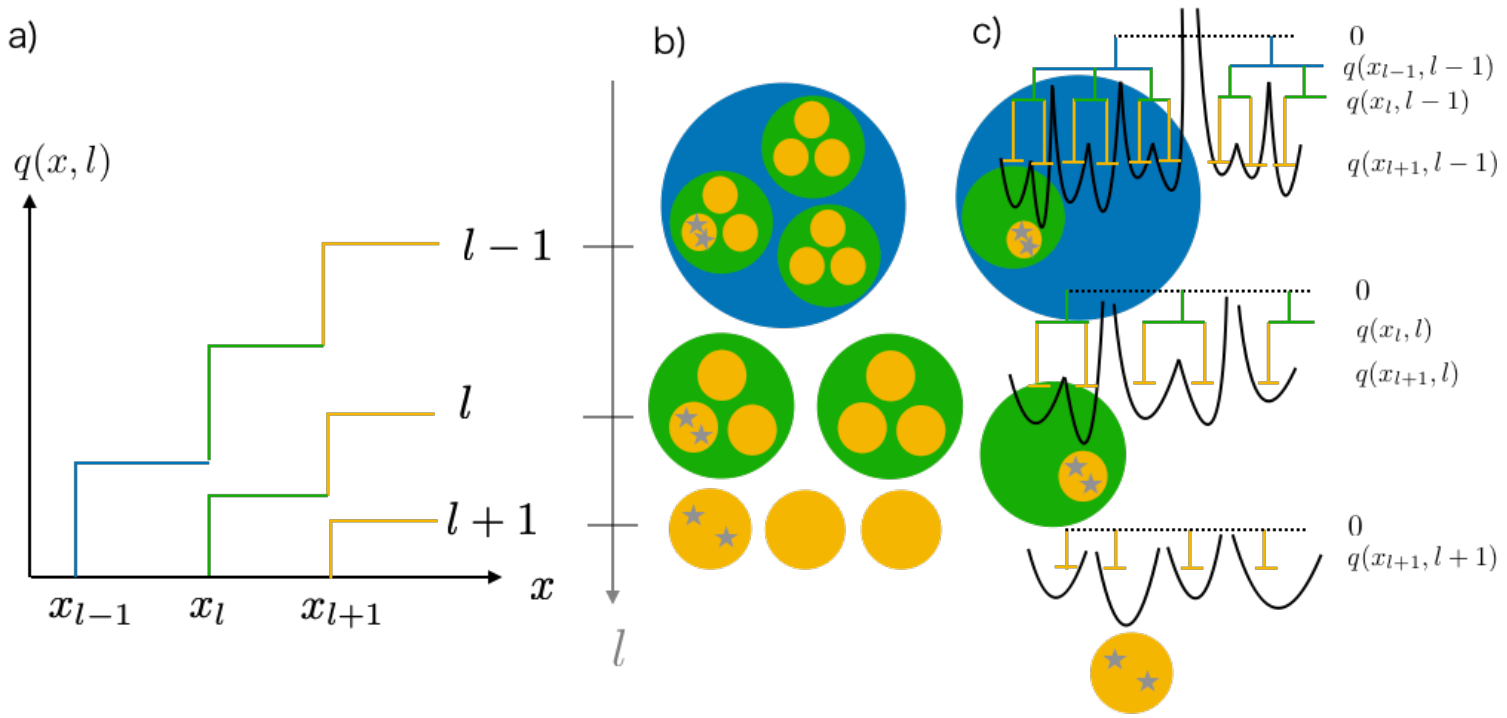

Figure 10: River terrace like glass order parameter and its implications: a) schematic picture of the river terrace like glass order parameter $q(x)$ (or $Q(x)$ ) b) hierarchical clustering of replicas c) schematic free-energy landscape and trees representing ultra-metric organization of overlaps between meta-stable states.

suggests that basic hierarchical structure of the overlap and that of the free-energy landscape is preserved across the glass phases at different layers. For example, consider again two replicas, i. e. two independent machines subjected to the same inputs/outputs represented by two 'stars' in the panels b) and c). For a given level, say at $n$-th level of the hierarchy, associated with the step at $x_{n}$, the probability that the two replicas sit in the common valley at level $n$ is $1-x_{n}$ which is the same at all layers up to the boundary $l=n, n-1, n-2, \ldots, 2,1$. It is tempting to speculate that these features have important consequences on learning in deep neural networks. We discuss possible implications of these in the last section.

\subsection{Fluctuating boundary}

In order to obtain further insights, we next analyze the case of fluctuating boundary: spin configuration on the boundaries are allowed to fluctuate during learning following certain probability distributions. Here we consider cases such that the overlap distribution of the spins on the input layer $(l=0)$ exhibit hierarchical, structure as parametrized in the form of the Parisi's matrix Eq. (28) (Fig. 11). There are several motivation for this analysis:

- The perturbation may provide some hints on the stability of the characteristic freeenergy landscape of the DNN we found above.

- It is natural to consider the case that input data fluctuates during learning as it happens in the standard Stochastic Gradient Descent (SGD) algorithms [1].

- Real data may not be totally random. It is natural to consider the possibility that input data set itself has a certain 'hidden' hierarchical structure, as ultra-metricity [14] is ubiquitous in nature. 


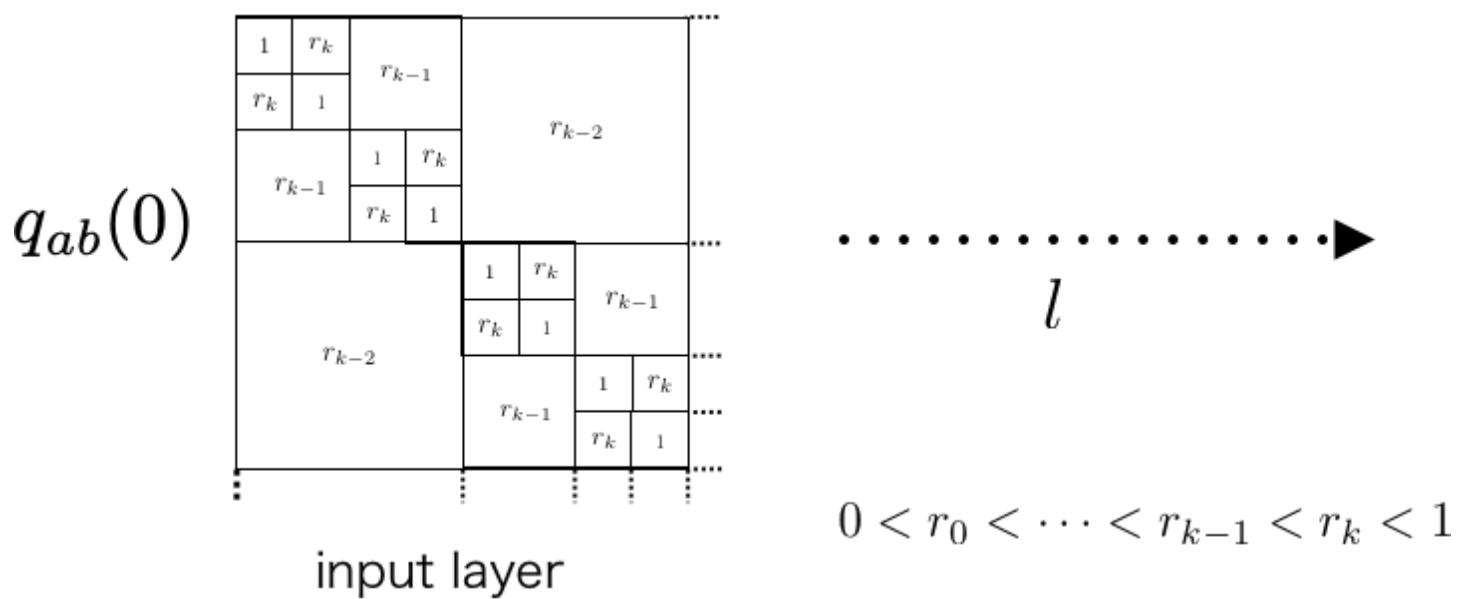

Figure 11: Fluctuating input layer with hierarchical overlap structure

\subsubsection{One RSB type boundary}

Here we consider the simplest case of '1RSB'.

$$
q_{i}(0)= \begin{cases}r & m_{i}<x_{\text {input }} \\ 1 & m_{i}>x_{\text {input }}\end{cases}
$$

This means the system is subjected to a slightly different input data, with overlap $0<r<1$ with respect the original input data, for some small probability $x_{\text {input }}$.

It can be seen in Fig. 12 that the effect of the perturbation is strong only at $x<x_{\text {input }}$. This means that the the hierarchical organization in the solution space has a certain degree of robustness.

\subsubsection{Full RSB type boundary}

Let us next consider 'full RSB' case. More specifically we consider the simplest full RSB structure in the input layer,

$$
q_{i}(0)=\min \left(a m_{i}, 1\right)
$$

with a certain constant $a>0$. Thus $q(x, 0)$ function consists of two parts: 1$)$ 'continuous part' $q(x, 0)=a x$ with slope $a$ in the interval $0<x<1 / a$ and 2) 'plateau' $q(x, 0)=q_{\mathrm{EA}}(0)=1$ in the interval $1 / a<x<1$.

We analyze the saddle point solutions numerically as before (see sec. B.3.3). In the following we present results using $k=100$ step RSB and the depth of the system $L=20$. We chose $1 / a=0.8$. As shown in Fig. 13, the glass phase grows increasing $\alpha$ much as in the case of quenched (RS) boundary condition. We limit ourselves to $\alpha$ such that $\xi_{\text {glass }}(\alpha)<L / 2$ so that the we have a liquid phase at the center of the system. In this circumstance the boundary condition on the other side $q_{a b}(L)$ is irrelevant.

A remarkable feature of the resultant glass order parameter is that the hierarchical structure put in the input propagate into the interior of the network preserving its basic structure. 

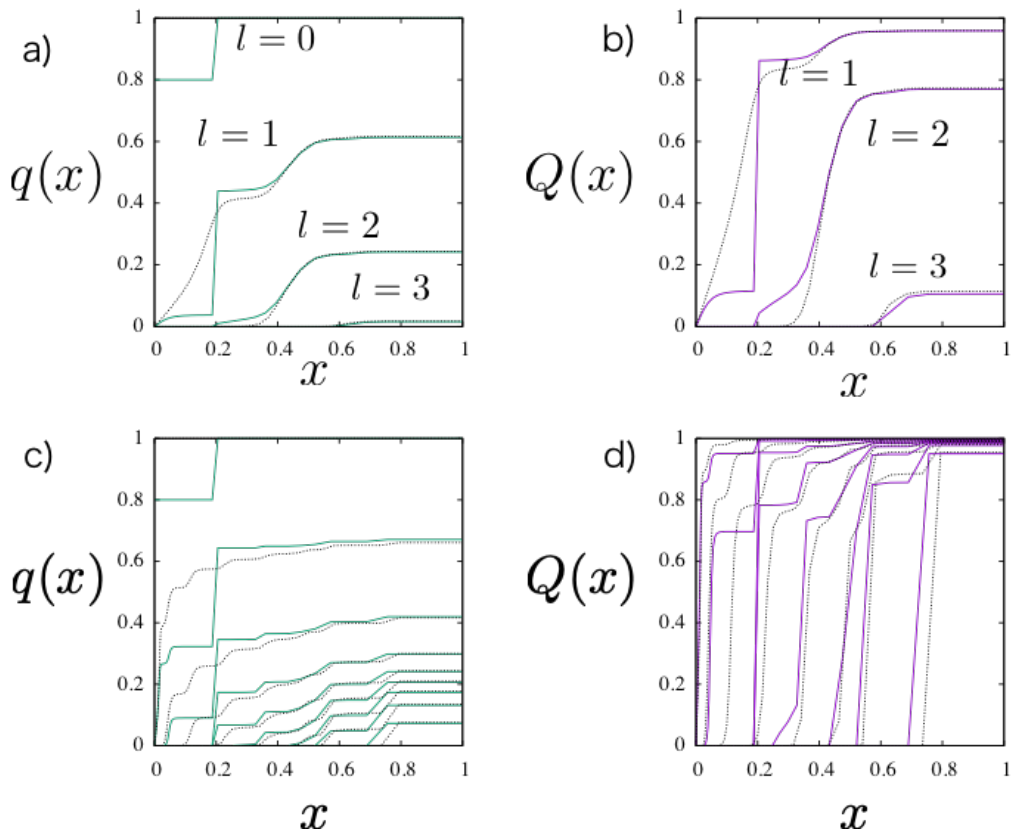

Figure 12: Glass order parameters with 1 RSB input. (solid lines) Here $L=20, x_{\text {input }}=0.2$ and $r=0.8$ for the solid lines and $x_{\text {input }}=0.2 \mathrm{a}$ ),b) $\alpha=50 \mathrm{c}$ ),d) $\alpha=4000$. (doted lines) the glass order parameters with frozen boundary.

Numerical solution suggests that the $q(x, l)$ function at a given layer $l$ consists of three parts: 0) $q(x, l)=0$ for some interval $\left.0<x<x_{l} 1\right)$ 'continuous part' $q(x, l)=a\left(x-x_{l}\right)$ in the interval $x_{l}<x<1 / a$ with the same slope $a$ as in the input 2) 'plateau' $q(x, l)=q_{\mathrm{EA}}(l)=1-a x_{l}$ in the last interval $1 / a<x<1$ as in the input. Correspondingly the overlap distribution function $P(q)=d x(q) / d q$ becomes,

$$
P(q, l)=x_{l} \delta(q)+\frac{1}{a}+\left(1-\frac{1}{a}\right) \delta\left(q-\left(1-a x_{l}\right)\right)
$$

which consists of three parts: 0 ) delta peak at $q=01$ ) constant part with height $1 / a$ in the interval $x_{l}<x<1 / a$ as in the input 2) delta peak at $q=q_{\mathrm{EA}}(l)$.

Going deeper into the interior increasing $l$, we find $x_{l}$ grows and $q_{\mathrm{EA}}(l)=1-a x_{l}$ decreases. We can regard this as a kind of 'renormalization' of the input data : the embedded overlap structure at low overlaps in the input data are progressively renormalized into $q=0$ sector, keeping only the hierarchical structure at higher overlaps. This means that a DNN works naturally as a machine for renormalization, i. e. classifications and feature detections.

\section{Teacher-student setting}

Now let us turn to analyze in detail the teacher-student setting introduced in sec. 2.2.2 by the replica theory using the ansatz explained in sec. 3.3.2. 

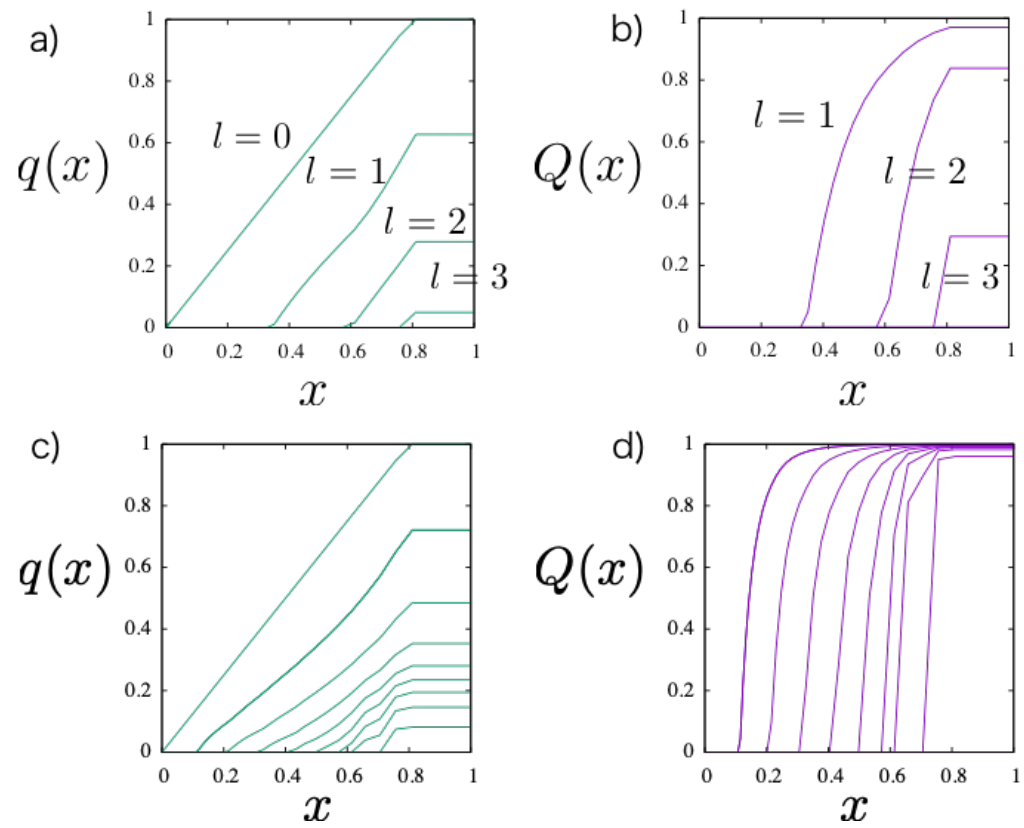

Figure 13: Glass order parameters with full RSB input. Here $L=20$ and $1 / a=0.8$. a),b) $\alpha=50$ c),d) $\alpha=4000$.

\subsection{Training}

Since we are limiting ourselves to the Bayes optimal case, it is sufficient to consider the replica symmetric $(k=0)$ ansatz so that the Nishimori condition holds [8,25], which read in the present system,

$$
r(l)=q_{0}(l) \quad R(l)=Q_{0}(l) .
$$

In Fig. 14 we show the spatial profile of the solutions obtained at various $\alpha=M / N$. Remarkably the spatial profile of the order parameters are very similar to those of random inputs/outputs (See Fig. 7). This is again due to successive layer-by-layer, 2nd order phase transitions which start from the boundaries. The overlap of the student machine with respect to the teacher machine grow from the boundary and the penetration depth grows again as

$$
\xi_{\text {teacher-student }} \propto \ln \alpha \text {. }
$$

The central part of the student machine remains de-correlated with respect to the teacher machine if the system is deep enough.

The result is somewhat paradoxical. On one hand, it is reasonable that the central part of a deep enough student machine remains flexible because the parameter space is huge. But on the other hand, if the central part remains really random, how can the student machine actually develop some finite overlap with respect to the teacher machine at the opposite ends disconnected by the liquid phase in between? Our analysis for the case of the random inputs/outputs would suggest otherwise: the student machine should not be able to pick up the minima planted by the teacher machine correctly hidden in the ocean of many (wrong) minima, all of which correctly satisfy the constraints on the input and output boundaries. 
A scenario to resolve the apparent paradox is the following. We note that each step of the layer-by-layer transition is a 2 nd order transition. Then the non-trivial saddle point solution we obtained here can be selected if some weak symmetry breaking field is present in the system. Most likely scenario is that some weak correlation between the teacher and student machines of order, say $O(\log (N) / N)$ which do not contribute the order parameter Eq. (11) in the limits $N, M \rightarrow \infty$ (with fixed $\alpha=N / M$ ), remain in the central part of the system and plays the role of the symmetry breaking field: the free-energy of the selected state will be lowered by an amount of order $O(\log (N))$ with respect to other low lying states. Such logarithmic correction naturally arise by integrating out fluctuation of the order parameters around the saddle point. Since replica symmetry holds in the present case, all eigenvalues of the stability matrix (Hessian matrix) around the saddle point are positive so that the saddle point integrations are well defined. We leave detailed analysis of the correction term for future studies.
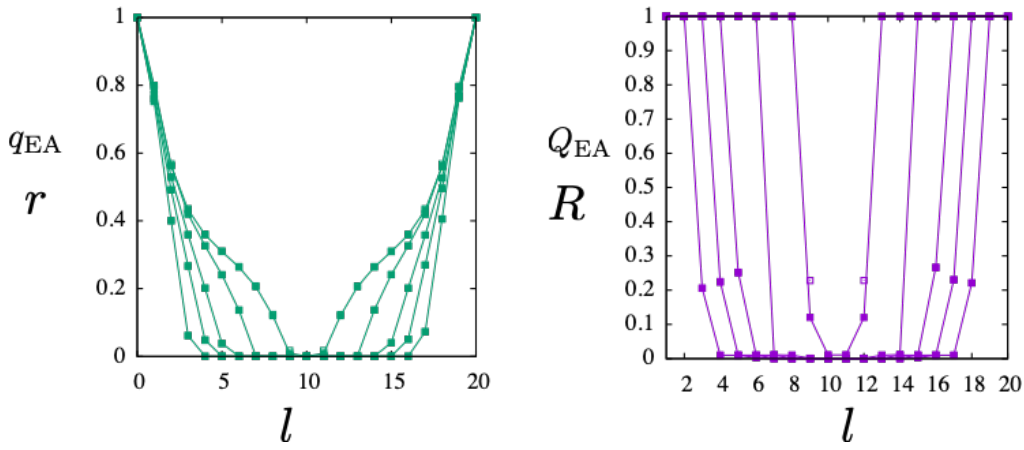

Figure 14: The spatial profile of the EA order parameters $q_{\mathrm{EA}}(l), Q_{\mathrm{EA}}(l)$ and overlap with between the student and teacher machines $r(l), R(l)(\mathrm{RS}$ solution $k=0)$ at $1 / \alpha=$ $0.08,0.04,0.02,0.01,0.005$. Here $L=20$.

\subsection{Generalization}

To test the generalization property of the student machine, we should compare the output of the teacher and student machines with respect to a set of test data, i. e. a new set of input data which is not used for the training.

To simulate this process within our framework we consider the following : solve again the replica symmetric saddle point equations for the order parameters of the spins $q(l)$ and $r(l)(\leq q(l))$ for $l=1,2, \ldots, L$ but with the order parameters of the bonds $Q(l)=R(l)$ for $l=1,2, \ldots, L$ fixed as determined above. At the input boundary $l=0$ we again set $q(0)=r(0)=1$ because the teacher and student machines are subjected to a common set of input data of the test data. On the other hand, now the output of the student machine is not constrained. Thus we do not put the other constraint $q(L)=r(L)=1$ but treat $q(L)$ and $r(L)(\leq q(L))$ also as additional variational parameters.

By solving the equations we recovered the same solutions as before, which are shown in Fig. 14. The result implies that, within our theory, the performance of the student machine with respect to the test data is essentially the same as that for the training data. Thus the 
generalization ability becomes poor entering the central liquid region but rapidly improves going through the crystalline region. Again we emphasize that this solution makes sense only in the presence of the remnant symmetry breaking field penetrating the liquid phase.

\section{Simulations of learning}

Now we turn to discuss some numerical simulations to examine our theoretical predictions regarding the two scenarios: 1) the setting of a random constraint satisfaction problem with random inputs/outputs at boundaries (Sec 2.2.1) and 2) the setting of a statistical inference problem with teacher and student machines (Sec 2.2.2).

\subsection{Relaxational dynamics of a soft-core model with random inputs/outputs}

In sec. 4 we found theoretically that the free-energy landscape of the perceptron network subjected to random constraints on the boundaries exhibit spatially heterogeneous structure: it is very complicated close to the boundaries but very simple in the central part. This naturally implies that learning dynamics is also heterogeneous in space.

To examine the nature of the learning dynamics we perform Monte Carlo simulations of the multi-layer neural network with depth $L$, width $N$ and randomly quenched inputs/outputs spins. The effective Hamiltonian of the system Eq. (8) reads as,

$$
H=\sum_{\mu} \sum_{\mathbf{\square}} V\left(r_{\mathbf{\square}}^{\mu}\right) \quad r_{\mathbf{\square}}^{\mu} \equiv \sum_{i=1}^{N} \frac{J_{\mathbf{m}}^{i}}{\sqrt{N}} S_{\mathbf{\square}}^{\mu}(i) S_{\mathbf{\square}}^{\mu}
$$

For convenience for the simulation, we replace the hard-core potential Eq. (6) by a soft-core potential,

$$
V(h)=\epsilon h^{2} \theta(-h)
$$

where $\epsilon$ is the unit of energy. Note that the statistical mechanics of a system with the soft-core potential becomes the same as the hardcore potential in the zero temperature limit $k_{\mathrm{B}} T / \epsilon \rightarrow 0$, where $k_{\mathrm{B}}$ is the Boltzmann's constant and $T$ is the temperature, in the region where all the constraints are satisfied (SAT).

The dynamical variables are the $M$-component vector spins and bonds,

$$
\begin{aligned}
& S^{\mu} \quad(\mu=1,2, \ldots, M) \quad(\mathbf{\square}=1,2, \ldots, N L) \\
& J_{\mathbf{a}}^{i} \quad(i=1,2, \ldots, N) \quad(\mathbf{\square}=1,2, \ldots, N L)
\end{aligned}
$$

Each component of the spins only take Ising values \pm 1 while each of the bonds take continuous values. In order to satisfy the normalization condition Eq. (2) $\sum_{i=1}^{N}\left(J_{\mathbf{m}}^{i}\right)^{2}=N$, we assume that $J_{\mathbf{m}}$ follows a Gaussian distribution with 0 mean and variance 1 . We performed simple Metropolis updates of the dynamical variables at very low temperatures $T$ to simulate the relaxational dynamics. In a sense the finite temperature Monte Carlo dynamics mimic the 'stochastic' nature of the standard Stochastic Gradient Descent (SGD) algorithms used for training of DNNs [1]. To propose a new spin configuration for the Metropolis algorithm, first we select a spin component $S_{\boldsymbol{n}}^{\mu}$ randomly out of the $N \times L \times M$ possibilities and then flip it 
as $S_{\boldsymbol{n}}^{\mu} \rightarrow-S_{\boldsymbol{n}}^{\mu}$. To update the bond configuration, first we select a bond $J_{\boldsymbol{\square}}^{i}$ randomly out of the $N \times L \times N$ possibilities and then shifted its value as,

$$
J_{\mathbf{a}}^{i} \rightarrow \frac{J_{\boldsymbol{n}}^{i}+r x}{\sqrt{1+r^{2}}}
$$

where $x$ is a random number following the Gaussian distribution with zero mean and variance 1. We set $r=0.1$ in our simulations. Within 1 MCS (Monte Carlo Step), the unit step of the Monte Carlo simulation, we try updates of the spins $N \times L \times M$ times and updates of the bonds $N \times L \times N$ times.

At first the configurations of the frozen spins on the input $l=0$ and the output $l=L$ layers are generated randomly. The initial configurations of the mobile spins are bonds are also generated randomly. Then spins and bonds are updated using the Metropolis algorithm at a low temperature $T$. In our simulations we set $k_{\mathrm{B}} T / \epsilon=0.015$.

We measure the following time autocorrelation functions,

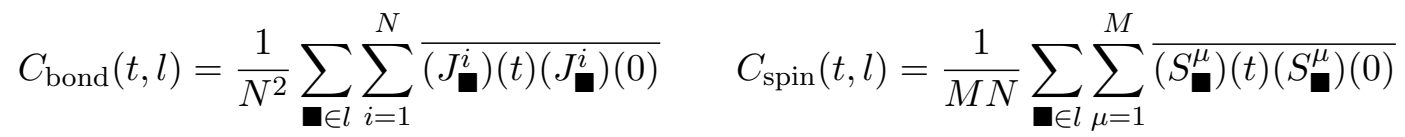

where $\mathbb{\square} \in$ stands for summation over the perceptrons within the $l$-th layer. As we noted in sec. 3.1 the system is symmetric under permutations of the perceptrons within the same layer. This does not matter here as long as we limit ourselves on the time scales where the correlation functions defined above remain positive. The overline $\cdots$ represents the average over different samples: the realization of the inputs/outputs spins are chosen randomly for each sample. In the following we used 240 samples.

In the present simulations we chose the depth as $L=5,10$, the width as $N=20$ and the number of the patterns of data as $M=100,200$, which means $\alpha=M / N=5,10$. In Fig. 15 we show data of the time autocorrelation functions of the spins and bonds.

From the two panels on the left-hand side in the low a), it can be seen that the dynamics is actually faster in the center and slower close to the boundaries as we expected. However in the semi-logarithmic plots on the right-hand side it can be seen that the dynamics is actually complicated: the dynamics is slow at shorter time scales at all layers which crossovers to faster dynamics at longer time scales. The difference of the speed of the relaxations at different layers is prominent in the longer times scales. The result suggests that the slow dynamics at the shorter time scales reflect the glassy aspect of the free-landscape and the truncation of the slow dynamics at longer time scales reflect the presence of the liquid phase at the center. It is interesting to note that similar truncation of the slow dynamics has been observed in a study of SGD dynamics in DNNs [18].

From the panels in the low b), it can be seen that the dynamics become slower as the strength of the constraints $\alpha$ increases. This is consistent with the theoretical expectation that the system becomes more glassy with larger $\alpha$.

From the panels in the low c), it can be seen that the depth $L$ do not change the slow dynamics at shorter time scales but changes the truncation of the slow dynamics. Relaxation at the longer time scale is apparently faster in deeper system. Interestingly this happens even in the layer just next to the boundaries. Presumably this implies that deeper system is more flexible in the center and the fast dynamics at the center assists the relaxation of the whole system. 
To summarize the numerical observation of the relaxations is qualitatively consistent with the theoretical prediction which implies spatially heterogeneous dynamics. However it should be noted that the dynamics is not symmetric with respect to the change of input and output sides. This is not captured by our theory because of the Gaussian approximation.

\subsection{Learning by a binary perceptron in a teacher-student setting}

Finally let us discuss results of some numerical simulations of the teacher-student setting which we studied theoretically in sec. 5. The teacher and student machines have exactly the same structures. For simplicity we use the binary perceptron model [26,27] in which the synaptic weights in Eq. (1) are restricted to take binary (Ising) values $J_{\mathbf{n}}^{k}= \pm 1$.

Some details of the simulations are listed below

- Teacher-machine

For the teacher machine we consider networks with $L=4,6$ and $N=4$. For each sample of the teacher machine, $N \times L$ values of the bonds $\left(J_{\mathbf{n}}^{k}\right)_{\text {teacher }}= \pm 1$ are generated randomly with no bias. Given a set of input data $\left.S_{0, i}^{\mu}\right)= \pm 1(i=1,2, \ldots, N),(\mu=$ $1,2, \ldots, M)$, it produces a set of corresponding output data $\left(S_{L, i}^{\mu}\right)_{\text {teacher }}= \pm 1(i=$ $1,2, \ldots, N),(\mu=1,2, \ldots, M)$.

Each input data $S_{0, i}^{\mu}= \pm 1$ is also generated randomly without bias. Two independent sets of inputs/outputs data, both with $M$ patterns, are prepared. One set is used to train the student machine and the other set is used to test the student machine.

- Student-machine

Student machine has the same architecture as the teacher machine. We train the student machine by performing a greedy Monte Carlo simulation which attempts to minimize a loss function,

$$
\mathcal{L}=\sum_{\mu=1}^{M} \sum_{i=1}^{N}\left(\left(S_{L, i}^{\mu}\right)_{\text {teacher }}-\left(S_{L, i}^{\mu}\right)_{\text {student }}\right)^{2} .
$$

where $\left(S_{L, i}^{\mu}\right)_{\text {teacher }}$ is the output of the teacher-machine. It goes as the following. 0) Initial values of the bonds $\left(J^{k}\right)_{\text {student }}$ are generated randomly with no bias. 1) At each micro-step of the simulation, one bond $\left(J_{\mathbf{n}}^{k}\right)_{\text {student }}$ out of all bonds of the student machine is selected. 2) If a flip $\left(J_{\mathbf{n}}^{k}\right)_{\text {student }} \rightarrow-\left(J_{\mathbf{n}}^{k}\right)_{\text {student }}$ lowers the loss function, the the flip is accepted and otherwise rejected. 3) the micro-step 1)-2) is repeated $N \times L$ times for one MCS. 4) Repeat 3) until $\mathcal{L}=0$ is reached. If it is not reached within $10^{6}$ MCS, we restart the whole process from 0 ).

- Training and test

For training, the set of inputs/outputs data prepared for the training is given to the student machine and we train the student machine using the greedy Monte Carlo algorithm as explained above. We examine the quality of the student machine after the training by computing the overlap of the bonds $R(l)$ and spins $r(l)$ at each layer $l$ (see below), between the teacher and student machines.

To test the generalization ability of the student machine, we use the other set of inputs/outputs data. We examine the performance of the student machine (now its bonds 
are fixed) by computing the overlap of the spins between the teacher and student machines, which we denote as $r_{\text {test }}(l)$.

- Overlap between the teach and student machines

We define the overlaps between the teacher and student machines as

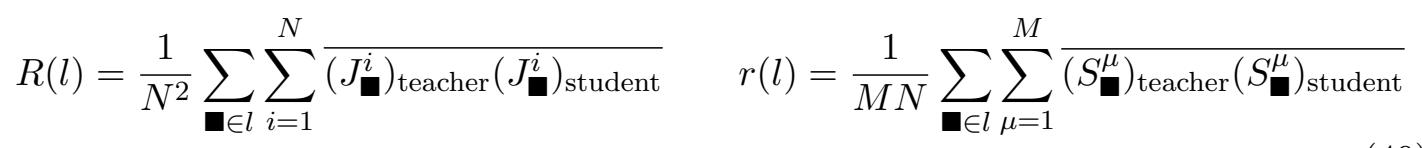

where $\mathbb{\square} \in l$ stands for summation over the perceptrons within the $l$-th layer. The overline $\cdots$ represents the average over different samples: both the realization of the teacher-machine and the input data are chosen randomly for each sample. In the following we used $O\left(10^{3}\right)$ samples.

As we noted in sec. 3.1 the system is symmetric under permutations of the perceptrons - within the same layer. This matters here because we are performing static sampling where permuted configurations should appear with the same statistical weights. In order to remove this unwanted symmetry, we removed $20 \%$ of the bonds randomly in each layer, but exactly in the same way, from both the teacher and student machines.

In Fig. 16 we show the result of the simulations where we used systems with $L=4$ and $N=4$ with $M=4,8,12,16$ so that $\alpha=M / N=1,2,3,4$. Thus the system is surely over-parametrized. The panels in the lows a) and b) show bond and spin overlaps after the training. As one can see in the two panels on the low a), the bond overlap $R(l)$ is large and increases with $\alpha$ on the two layers just next to the inputs/outputs boundaries while it remains very small in the middle. Correspondingly it can be seen in the two panels on the low b) that the spin overlap $r(l)$ also show similar tendencies.

As shown in the two panels on the low c), the spin overlap $r_{\text {test }}(l)$ for the test data is almost the same as that for the training data $r(l)$ shown on the low b), except for the value at the very end $r_{\text {test }}(L)$. Note that $r(L)=1$ by construction of the training while $r_{\text {test }}(L)$ can vary during test. It can be seen that $r_{\text {test }}(L)$ clearly increases with $\alpha$ proving the generalization ability of the student machine in spite of the fact that the system is over-parametrized.

Remarkably these features are qualitatively consistent with the theory discussed in sec. 5 . which predicts that finite overlaps between the teacher and student machines emerge from the boundaries and the saddle point solutions in the training and test stages are essentially the same. However it should be noted again that the result is not symmetric with respect to the change of input and output sides. This is not captured by our theory because of the Gaussian approximation.

As we pointed out in sec. 5 there must be some symmetry breaking field connecting the opposite sides of the system to enable learning in such over-parametrized machines with 'liquid' in the center. Indeed it can be seen that, although small, finite positive overlap remains also in the central area. In Fig 16 we show width $N$ dependence of the bond and spin overlaps after the training. It can be seen clearly that the overlaps decreases with increasing width $N$ for fixed $\alpha=N / M$. This consistent with the expectation that the small positive overlap remaining in the center is a finite width $N$ correction term. 


\section{Conclusion and outlook}

\subsection{Conclusion}

In the present paper we constructed a statistical mechanical theory for the solution space of a deep neural network of depth $L$, width $N$ subjected to $M=\alpha N$ patterns of data using the internal representation, based on the replica method within a Gaussian approximation in the limit $N, M \rightarrow \infty$ with fixed $\alpha$. We studied two scenarios : 1) the case of random inputs/outputs, which is a random constraint satisfaction problem and 2) a teacher-student setting, which is a statistical inference problem. In addition, we performed two sets of simulations to examine the theoretical predictions on 1) and 2).

The main outcome of the theory is the prediction of the heterogeneous spatial profile of states inside the layered network : 1) 'glass-liquid-glass' in the case of the random inputs/outputs 2) 'crystal-liquid-crystal' in the case of the teacher-student setting. The thickness of the glass/crystal phase grows as $\xi \propto \ln \alpha$. Moreover, in the case of the setting 1) random inputs/outputs, even the pattern of the replica symmetry breaking (RSB) varies in space : it is most complex close to the boundaries with $k$ (+continuous)-RSB, which becomes $k-1$ (+continuous)-RSB in the next layer, ... down to a replica symmetric (0 RSB) state in the central part. The hierarchical structures in different layers are synchronized. We argued that small the positive overlap can remain in the liquid phase as a finite $N$ correction and plays the role of symmetry breaking field connecting the opposite side of the system.

There are some weak points in our theory. First of all, the Gaussian approximation does not capture the asymmetry inherent in the feed-forward network. Second, our theory assumes thermodynamic limit $N, M \rightarrow \infty$ (with $\alpha=M / N$ fixed) while real networks have finite width $N$ so that 'phase transitions' we found here become crossovers. Nonetheless we believe our results are still provide useful guidelines to understand real DNNs.

For 1), we performed simulation of the relaxational dynamics. We found heterogeneous dynamics which is fast in the center and slow closer to the boundaries in agreement with the theory. For 2) we simulated learning in a teacher-student setting. We found heterogeneous learning and generalization as expected from the theory. The presence of remnant bias in the center is found, which appear to be compatible with the interpretation as due to a finite width $N$ correction.

To summarize, both the theory and the simulation suggest spatially heterogeneous freeenergy landscape in the over parametrized DNNs. We speculate that this is responsible for the efficiency of DNNs in three respects:

a) The presence of the liquid phase in the center facilitates the equilibration (learning) of the whole system. The sclae $\xi \propto \ln \alpha$ may be considered as a useful guide for the choice of the depth $L$, which is a key hyper-parameter.

b) In spite of the over-parametrization the system can generalize well because of the presence of the crystalline phase close to the boundaries. Weak bias which remain in the liquid phase as a finite width $N$ correction plays the crucial role of symmetry breaking field to select the hidden crystalline state out of many glass states.

c) Hierarchical free-energy landscapes with ultra-metric structure has been known in glass physics since the discovery of replica symmetry breaking [13,14]. Our theory suggests that it evolves in space in DNNs progressively from complex to simple ones going deeper 
into the interior. This implies DNN naturally have the power to do renormalization of the data, i. e. classifications and feature detections.

\subsection{Outlook}

Here we list some suggestions for further investigations. First of all, it will be very interesting to perform extensive numerical simulations with realistic algorithms and realistic large-scale data to examine our predictions. Second, more detailed theoretical/numerical analysis of the remnant bias field in the liquid phase will be interesting. Third, it is important to asses carefully the nature of the Gaussian approximation we made in the present paper and try to go beyond it to capture more faithfully the directional character of the feed-forward network. Lastly it is natural to extend the present work limited to the simplest setting to more general ones regarding the activation function, architecture, and learning method. Our work may also have implications on various complex systems with heterogeneity, including ultra-stable glass $[28,29]$, gene regulatory networks [30-32] and allosteric systems [33-35].

\section{Acknowledgments}

We thank Giulio Biroli, Silvio Franz, Sungmin Hwang, Macoto Kikuchi, Kota Mitsumoto, Tomoyuki Obuchi, Pierfrancesco Urbani, Lenka Zdeborová and Francesco Zamponi for useful discussions. This work was supported by KAKENHI (No. 19H01812) from MEXT, Japan. Numerical analysis in this work has been done using the supercomputer systems OCTOPUS and SX-ACE at the Cybermedia Center, Osaka University. 
a)
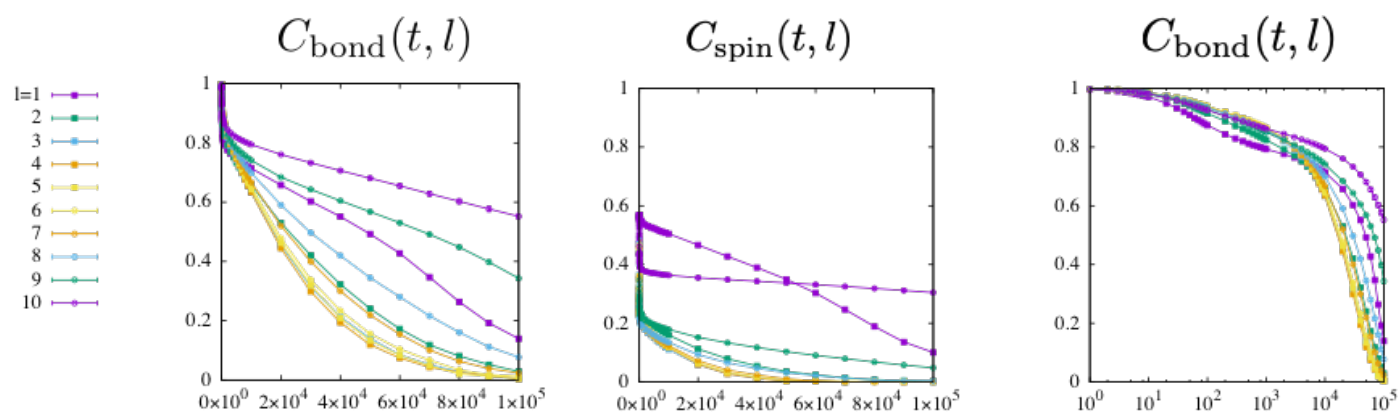

b)
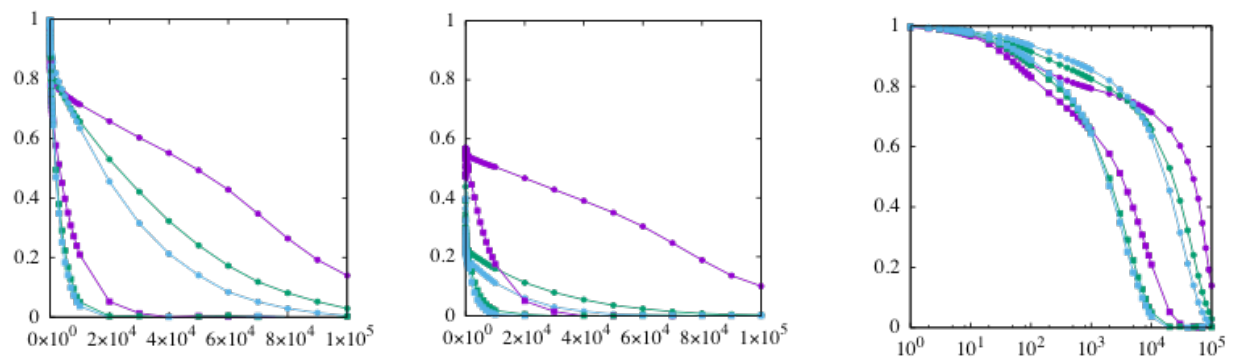

c)
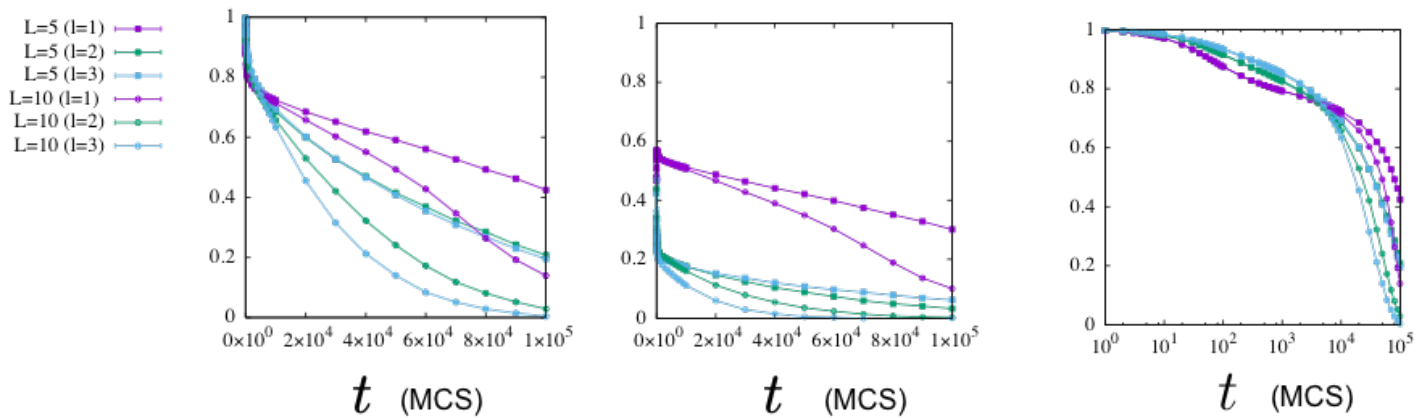

Figure 15: Relaxation of auto-correlation functions of the bonds $C_{\text {bond }}(t, l)$ and spins $C_{\text {spin }}(t, l)$. The two columns on the left hand side display correlation functions of the bonds and spins plotted against time $t$. The column on the right hand side display the same data of $C_{\text {bond }}(t, l)$ against logarithmic time scale. In the 1st low a) data at different layers $l=1,2, \ldots, 10(L=10, \alpha=10)$ are shown. In the 2 nd low b) data for $\alpha=5$ and 10 $(L=10)$ are shown. In the 3rd low c) data obtained with different depth $L=5$ and 10 $(\alpha=10)$ are shown. Error bars are smaller than the size of the symbols. 

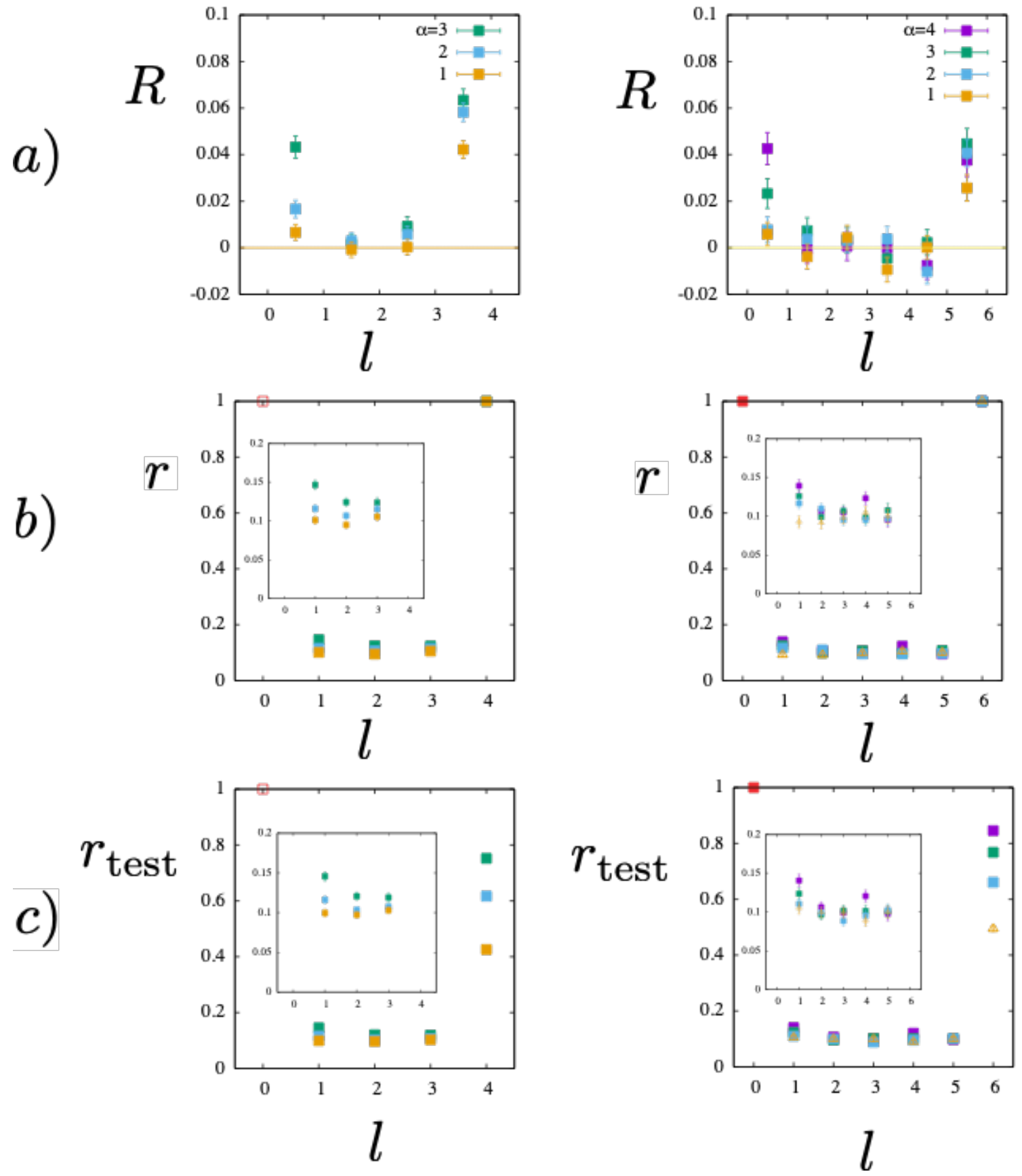

Figure 16: Overlap between the teacher and student machines. a) overlap of the bonds for training data , b) the same as a) but for the spins and c) overlap of the spins for the test data. The left column is for $L=4, N=4$ and $\alpha=N / M=1,2,3$. The right column is for $L=6, N=4$ and $\alpha=N / M=1,2,3,4$. Note that $r(0)=r(L)=r_{\text {test }}(0)=1$ by construction of the protocol. On the other hand, the value of $r_{\text {test }}(L)$ represent the generalization ability of the student-machine. 
a)

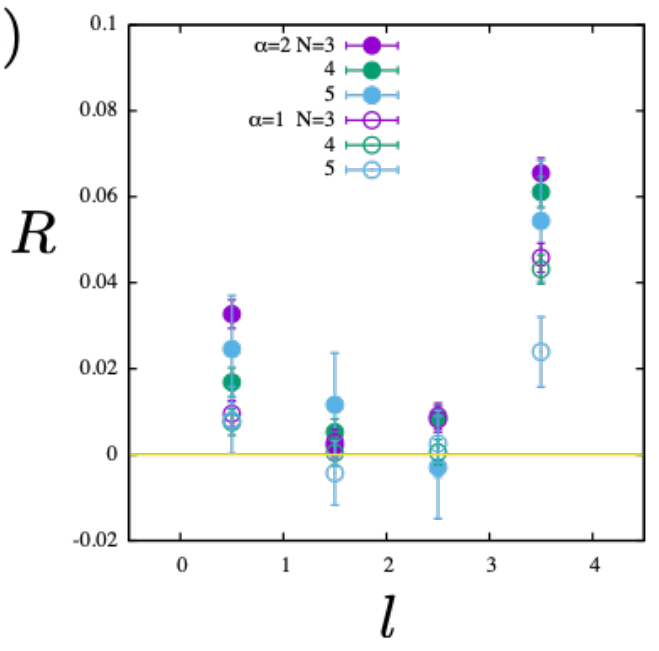

b)

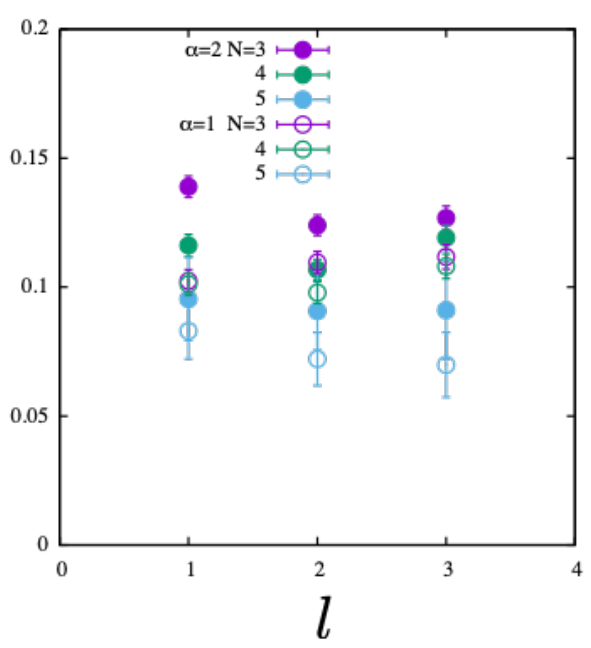

c)

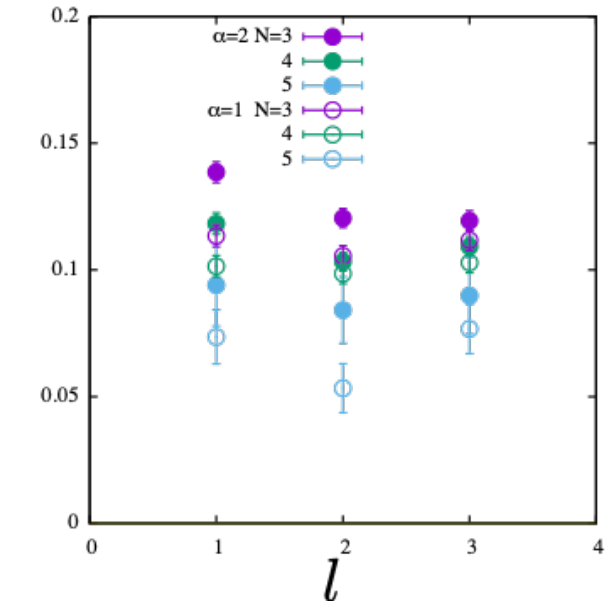

Figure 17: Width $N$ dependence of the a) bond and b) spin overlap for the training data, and c) spin overlap for the test data with $\alpha=M / N=1,2$ for $N=3,4,5$. Here $L=4$. 


\section{References}

[1] Y. LeCun, Y. Bengio and G. Hinton, Deep learning, nature 521(7553), 436 (2015).

[2] G. Cybenko, Approximation by superpositions of a sigmoidal function, Mathematics of control, signals and systems 2(4), 303 (1989).

[3] C. Zhang, S. Bengio, M. Hardt, B. Recht and O. Vinyals, Understanding deep learning requires rethinking generalization, arXiv preprint arXiv:1611.03530 (2016).

[4] G. Carleo, I. Cirac, K. Cranmer, L. Daudet, M. Schuld, N. Tishby, L. Vogt-Maranto and L. Zdeborová, Machine learning and the physical sciences, arXiv preprint arXiv:1903.10563 (2019).

[5] E. Gardner, The space of interactions in neural network models, Journal of physics A: Mathematical and general 21(1), 257 (1988), doi:10.1088/0305-4470/21/1/030.

[6] E. Gardner and B. Derrida, Three unfinished works on the optimal storage capacity of networks, Journal of Physics A: Mathematical and General 22(12), 1983 (1989).

[7] M. Mezard and A. Montanari, Information, physics, and computation, Oxford University Press (2009).

[8] L. Zdeborová and F. Krzakala, Statistical physics of inference: Thresholds and algorithms, Advances in Physics 65(5), 453 (2016), doi:10.1080/00018732.2016.1211393.

[9] L. Berthier and G. Biroli, Theoretical perspective on the glass transition and amorphous materials, Reviews of Modern Physics 83(2), 587 (2011), doi:10.1103/RevModPhys.83.587.

[10] P. Charbonneau, J. Kurchan, G. Parisi, P. Urbani and F. Zamponi, Fractal free energy landscapes in structural glasses, Nature communications 5, 3725 (2014), doi:10.1038/ncomms4725.

[11] A. Engel and C. Van den Broeck, Statistical mechanics of learning, Cambridge University Press (2001).

[12] G. Parisi, Infinite number of order parameters for spin-glasses, Physical Review Letters 43(23), 1754 (1979), doi:10.1103/PhysRevLett.43.1754.

[13] M. Mézard, G. Parisi and M. A. Virasoro, Spin glass theory and beyond, World Scientific, Singapore (1987).

[14] R. Rammal, G. Toulouse and M. A. Virasoro, Ultrametricity for physicists, Reviews of Modern Physics 58(3), 765 (1986).

[15] S. Franz and G. Parisi, The simplest model of jamming, Journal of Physics A: Mathematical and Theoretical 49(14), 145001 (2016), doi:10.1088/1751-8113/49/14/145001.

[16] S. Franz, G. Parisi, M. Sevelev, P. Urbani and F. Zamponi, Universality of the sat-unsat (jamming) threshold in non-convex continuous constraint satisfaction problems, SciPost Physics 2(3), 019 (2017), doi:10.21468/SciPostPhys.2.3.019. 
[17] H. Yoshino, Disorder-free spin glass transitions and jamming in exactly solvable mean-field models, SciPost Phys. 4, 40 (2018), doi:10.21468/SciPostPhys.4.6.040.

[18] M. Baity-Jesi, L. Sagun, M. Geiger, S. Spigler, G. B. Arous, C. Cammarota, Y. LeCun, M. Wyart and G. Biroli, Comparing dynamics: Deep neural networks versus glassy systems, arXiv preprint arXiv:1803.06969 (2018).

[19] S. Franz, S. Hwang and P. Urbani, Jamming in multilayer supervised learning models, arXiv preprint arXiv:1809.09945 (2018).

[20] M. Geiger, S. Spigler, S. d'Ascoli, L. Sagun, M. Baity-Jesi, G. Biroli and M. Wyart, Jamming transition as a paradigm to understand the loss landscape of deep neural networks, Physical Review E 100(1), 012115 (2019).

[21] R. Monasson and R. Zecchina, Weight space structure and internal representations: a direct approach to learning and generalization in multilayer neural networks, Physical review letters $\mathbf{7 5}(12), 2432$ (1995).

[22] S. Franz and G. Parisi, Recipes for metastable states in spin glasses, Journal de Physique I 5(11), 1401 (1995), doi:10.1051/jp1:1995201.

[23] E. Gardner, Spin glasses with p-spin interactions, Nuclear Physics B 257, 747 (1985), doi:10.1016/0550-3213(85)90374-8.

[24] J. Kurchan, G. Parisi, P. Urbani and F. Zamponi, Exact theory of dense amorphous hard spheres in high dimension. II. The high density regime and the gardner transition, J. Phys. Chem. B 117, 12979 (2013).

[25] H. Nishimori, Statistical physics of spin glasses and information processing: an introduction, 111. Clarendon Press (2001).

[26] E. Gardner and B. Derrida, Optimal storage properties of neural network models, Journal of Physics A: Mathematical and general 21(1), 271 (1988).

[27] W. Krauth and M. Mézard, Storage capacity of memory networks with binary couplings, Journal de Physique 50(20), 3057 (1989).

[28] S. F. Swallen, K. L. Kearns, M. K. Mapes, Y. S. Kim, R. J. McMahon, M. D. Ediger, T. Wu, L. Yu and S. Satija, Organic glasses with exceptional thermodynamic and kinetic stability, Science 315(5810), 353 (2007).

[29] T. Pérez-Castañeda, C. Rodríguez-Tinoco, J. Rodríguez-Viejo and M. A. Ramos, Suppression of tunneling two-level systems in ultrastable glasses of indomethacin, Proceedings of the National Academy of Sciences 111(31), 11275 (2014).

[30] A. Wagner, Evolution of gene networks by gene duplications: a mathematical model and its implications on genome organization, Proceedings of the National Academy of Sciences 91(10), 4387 (1994).

[31] A. Wagner, Does evolutionary plasticity evolve?, Evolution 50(3), 1008 (1996).

[32] S. Nagata and M. Kikuchi, Emergence of cooperative bistability and robustness of gene regulatory networks, arXiv preprint arXiv:1907.12030 (2019). 
[33] J. Monod, J. Wyman and J.-P. Changeux, On the nature of allosteric transitions: a plausible model, J Mol Biol 12(1), 88 (1965).

[34] J. W. Rocks, N. Pashine, I. Bischofberger, C. P. Goodrich, A. J. Liu and S. R. Nagel, Designing allostery-inspired response in mechanical networks, Proceedings of the National Academy of Sciences 114(10), 2520 (2017).

[35] L. Yan, R. Ravasio, C. Brito and M. Wyart, Architecture and coevolution of allosteric materials, Proceedings of the National Academy of Sciences 114(10), 2526 (2017).

[36] M. Mézard and G. Parisi, Replica field theory for random manifolds, Journal de Physique I 1(6), 809 (1991), doi:10.1051/jp1:1991171.

[37] B. Duplantier, Comment on parisi's equation for the sk model for spin glasses, Journal of Physics A: Mathematical and General 14(1), 283 (1981), doi:10.1088/0305$4470 / 14 / 1 / 027$. 


\section{A Replicated free-energy}

Here we derive the free-energy free-energy functional of the replicated system Eq. (15)-Eq. (17) starting from Eq. (12), following similar steps as in [17].

\section{A.1 Evaluation of the entropic part of the free-energy}

We introduce 'local' order parameters [17],

$$
Q_{a b, \boldsymbol{\square}}=\frac{1}{N} \sum_{i=1}^{N}\left(J_{\mathbf{\square}}^{i}\right)^{a}\left(J_{\mathbf{\bullet}}^{i}\right)^{b} \quad q_{a b, \boldsymbol{\square}}=\frac{1}{M} \sum_{\mu=1}^{M}\left(S_{\mathbf{\bullet}}^{\mu}\right)^{a}\left(S_{\mathbf{\square}}^{\mu}\right)^{b}
$$

through the identities,

$$
\begin{aligned}
& 1=\int \prod_{a<b} d Q_{a b, \boldsymbol{\varpi}} d \epsilon_{a b, \boldsymbol{\varpi}} e^{i c \sum_{a<b}\left(\epsilon_{a b, \boldsymbol{\varpi}} Q_{a b, \boldsymbol{\varpi}}-c^{-1} \sum_{i=1}^{N}\left(J_{\mathbf{\bullet}}^{i}\right)^{a}\left(J_{\mathbf{\bullet}}^{i}\right)^{b}\right)} \\
& 1=\int \prod_{a<b} d q_{a b, \boldsymbol{\varpi}} d \varepsilon_{a b, \boldsymbol{\varpi}} e^{i M \sum_{a<b}\left(\varepsilon_{a b, \boldsymbol{\varpi}} q_{a b, \boldsymbol{\varpi}}-M^{-1} \sum_{\mu=1}^{M}\left(S_{\boldsymbol{\bullet}}^{\mu}\right)^{a}\left(S_{\boldsymbol{\bullet}}^{\mu}\right)^{b}\right)}
\end{aligned}
$$

by which we can write

$$
\begin{aligned}
& \prod_{a} \operatorname{Tr}_{\mathbf{J}_{\mathbf{\bullet}}^{a}} \cdots=\left(\prod_{a<b} \int_{-\infty}^{\infty} d\left(Q_{a b, \boldsymbol{\varpi}}\right)\right) e^{c s_{\mathrm{ent}, \mathrm{bond}}[\hat{Q} \mathbf{\square}]} \prod_{i=1}^{N}\langle\cdots\rangle_{J^{i}} \\
& \prod_{a} \operatorname{Tr}_{\mathbf{S}_{\mathbf{\square}}^{a}} \cdots=\left(\prod_{a<b} \int_{-\infty}^{\infty} d\left(q_{a b, \boldsymbol{\varpi}}\right)\right) e^{M s_{\mathrm{ent}, \mathrm{spin}}[\hat{q} \mathbf{\varpi}]} \prod_{\mu=1}^{M}\langle\cdots\rangle_{S^{\mu}}
\end{aligned}
$$

where we have performed integrations over $\epsilon_{a b}$ and $\varepsilon_{a b}$ by the saddle point method assuming $c \gg 1$ and $M \gg 1$. We find,

$$
\begin{aligned}
& s_{\text {ent,bond }}[\hat{Q}]=\sum_{a<b} \varepsilon_{a b}^{*} Q_{a b}+\ln \prod_{c=1}^{n} \operatorname{Tr}_{J^{c}} e^{-\sum_{a<b} \varepsilon_{a b}^{*} J^{a} J^{b}} \\
& \langle\cdots\rangle_{J^{i}}=\frac{\prod_{c=1}^{n} \operatorname{Tr}_{J^{c}} e^{-\sum_{a<b} \varepsilon_{a b}^{*}\left(J^{a}\right)^{\mu}\left(J^{b}\right)^{\mu}} \cdots}{\prod_{c=1}^{n} \operatorname{Tr}_{J^{c}} e^{-\sum_{a<b} \varepsilon_{a b}^{*}\left(J^{a}\right)^{\mu}\left(J^{b}\right)^{\mu}}}
\end{aligned}
$$

and

$$
\begin{aligned}
& s_{\text {ent,spin }}[\hat{q}]=\sum_{a<b} \epsilon_{a b}^{*} q_{a b}+\ln \prod_{c=1}^{n} \operatorname{Tr}_{S^{c}} e^{-\sum_{a<b} \epsilon_{a b}^{*} S^{a} S^{b}} \\
& \langle\cdots\rangle_{S^{\mu}}=\frac{\prod_{c=1}^{n} \operatorname{Tr}_{S^{c}} e^{-\sum_{a<b} \epsilon_{a b}^{*}\left(S^{a}\right)^{\mu}\left(S^{b}\right)^{\mu}} \cdots}{\prod_{c=1}^{n} \operatorname{Tr}_{S^{c}} e^{-\sum_{a<b} \epsilon_{a b}^{*}\left(S^{a}\right)^{\mu}\left(S^{b}\right)^{\mu}}}
\end{aligned}
$$

where $\epsilon_{a b}^{*}=\epsilon_{a b}^{*}(\hat{Q})$ and $\varepsilon_{a b}^{*}=\varepsilon_{a b}^{*}(\hat{q})$ are determined by

$Q_{a b}=\left.\frac{\prod_{c} \operatorname{Tr}_{J^{c}} e^{-\sum_{a<b} \varepsilon_{a b} J^{a} J^{b}} J^{a} J^{b}}{\prod_{c} \operatorname{Tr}_{J^{c}} e^{-\sum_{a<b} \epsilon_{a b} J^{a} J^{b}}}\right|_{\varepsilon_{a b}=\varepsilon_{a b}^{*}(\hat{q})} \quad q_{a b}=\left.\frac{\prod_{c} \operatorname{Tr}_{S^{c}} e^{-\sum_{a<b} \epsilon_{a b} S^{a} S^{b}} S^{a} S^{b}}{\prod_{c} \operatorname{Tr}_{S^{c}} e^{-\sum_{a<b} \epsilon_{a b} S^{a} S^{b}}}\right|_{\epsilon_{a b}=\epsilon_{a b}^{*}(\hat{Q})}$ 
The above equations imply in particular,

$$
\begin{aligned}
& \left\langle\left(J_{\mathbf{\square}}^{i}\right)^{a}\right\rangle_{J^{i}}=0 \quad\left\langle\left(J_{\mathbf{\square}}^{i}\right)^{a}\left(J_{\mathbf{\square}}^{j}\right)^{b}\right\rangle_{J^{i}}=Q_{a b} \delta_{i j} \\
& \left\langle\left(S_{\mathbf{\square}}^{\mu}\right)^{a}\right\rangle_{S^{\mu}}=0 \quad\left\langle\left(S_{\boldsymbol{\square}}^{\mu}\right)^{a}\left(S_{\mathbf{\square}}^{\nu}\right)^{b}\right\rangle_{S^{\mu}}=q_{a b} \delta_{\mu \nu}
\end{aligned}
$$

\section{A.1.1 Entropic part of the 'bonds'}

The bonds are continuous variables normalized as,

$$
\sum_{i=1}^{N}\left(J_{\mathbf{m}(i)}\right)^{2}=N
$$

which implies,

$$
Q_{a a}=1
$$

and

$$
S_{\text {ent,bond }}[\hat{Q}]=\frac{1}{2} \ln \operatorname{det} \hat{Q}
$$

\section{A.1.2 Entropic part of the 'spins'}

The spins are 'Ising spins'. The entropic part of the free-energy of the spins are [17],

$$
\begin{aligned}
s_{\text {ent,spin }}[\hat{q}] & =\sum_{a<b} \varepsilon_{a b}^{*} q_{a b}+\left.\ln e^{-\sum_{a<b} \varepsilon_{a b}^{*} \frac{\partial^{2}}{\partial h_{a} \partial h_{b}}} \prod_{a} 2 \cosh \left(h_{a}\right)\right|_{\left\{h_{a}=0\right\}} \\
& =\frac{1}{2} \sum_{a, b} \varepsilon_{a b}^{*} q_{a b}+\left.\ln e^{-\frac{1}{2} \sum_{a, b} \varepsilon_{a b}^{*} \frac{\partial^{2}}{\partial h_{a} \partial h_{b}}} \prod_{a} 2 \cosh \left(h_{a}\right)\right|_{\left\{h_{a}=0\right\}}
\end{aligned}
$$

with $q_{a a}=1$. Here we performed the spin trace formally as

$$
\begin{aligned}
& \operatorname{Tr}_{\mathbf{S}_{c}} e^{-\sum_{a<b} \varepsilon_{a b} S^{a} S^{b}}=e^{\frac{1}{2} \sum_{a} \epsilon_{a a}} \operatorname{Tr}_{\mathbf{S}_{c}} e^{-\frac{1}{2} \sum_{a, b} \varepsilon_{a b} S^{a} S^{b}}=\left.e^{\frac{1}{2} \sum_{a} \epsilon_{a a}} \operatorname{Tr}_{\mathbf{S}_{c}} e^{-\frac{1}{2} \sum_{a, b} \varepsilon_{a b} \frac{\partial^{2}}{\partial h_{a} \partial h_{b}} e^{\sum_{a} h_{a} S^{a}}}\right|_{\left\{h_{a}=0\right\}} \\
& =\left.e^{\frac{1}{2} \sum_{a} \epsilon_{a a}} e^{-\frac{1}{2} \sum_{a, b} \varepsilon_{a b} \frac{\partial^{2}}{\partial h_{a} \partial h_{b}}} \prod_{a} 2 \cosh \left(h_{a}\right)\right|_{\left\{h_{a}=0\right\}}
\end{aligned}
$$

For the integration over $\varepsilon_{a b}$, the saddle point $\varepsilon_{a b}^{*}=\varepsilon_{a b}^{*}[\hat{q}]$ is obtained formally as,

$$
q_{a b}=-\left.\left.\frac{\delta}{\delta \varepsilon_{a b}} \ln e^{-\frac{1}{2} \sum_{a, b} \varepsilon_{a b} \frac{\partial^{2}}{\partial h_{a} \partial h_{b}}} \prod_{a} 2 \cosh \left(h_{a}\right)\right|_{\left\{h_{a}=0\right\}}\right|_{\varepsilon_{a b}=\varepsilon_{a b}^{*}[\hat{q}]}
$$

\section{A.2 Evaluation of interaction part of the free-energy}

Now we wish to evaluate the partition function Eq. (12) using the tools developed above. To this end it is useful to consider the following properties. 
In Eq. (53) we notice that different spin components $\mu$ are decoupled in the average $\prod_{\mu}\langle\ldots\rangle_{S^{\mu}}$. Then we obtain the following cumulant expansion which will become very useful. For any observable $A=A\left(S^{\mu}\right)$ and writing $\langle\ldots\rangle_{S^{\mu}}=\langle\ldots\rangle$ for simplicity we find,

$$
\begin{aligned}
\ln \left\langle e^{\frac{1}{\sqrt{M}} \sum_{\mu=1}^{M} A_{\mu}}\right\rangle= & \sqrt{M}\left\langle A_{\mu}\right\rangle+\frac{1}{2 !}\left(\left\langle A_{\mu}^{2}\right\rangle-\left\langle A_{\mu}\right\rangle^{2}\right) \\
& +\frac{1}{3 ! \sqrt{M}}\left(\left\langle A_{\mu}^{3}\right\rangle-3\left\langle A_{\mu}^{2}\right\rangle^{2}\left\langle A_{\mu}\right\rangle+2\left\langle A_{\mu}\right\rangle^{3}\right)+\ldots
\end{aligned}
$$

Here we just used the fact that $\left\langle A^{\mu} A^{\nu}\right\rangle=\left\langle A^{\mu}\right\rangle\left\langle A^{\nu}\right\rangle$ holds for $\mu \neq \nu$. Thus in the $M \rightarrow \infty$, the lowest non-vanishing cumulant dominates the r.h.s. For instance if $\left\langle A_{\mu}\right\rangle=0$ and $\left\langle A_{\mu}^{2}\right\rangle \neq 0$, then $\lim _{M \rightarrow \infty} \ln \left\langle e^{\frac{1}{\sqrt{M}} \sum_{\mu=1}^{M} A_{\mu}}\right\rangle=\frac{1}{2 !}\left\langle A_{\mu}^{2}\right\rangle$. Note that the same property also holds for the averaging in the 'bond space' $\langle\ldots\rangle_{J^{i}}$ in Eq. (52).

\section{A.2.1 Original model}

Now the evaluation of the replicated Gardner volume Eq. (12). We find,

$$
\begin{aligned}
& \left\langle\left\langle\prod_{\mu, \boldsymbol{\square}, a} \exp \left[i \eta_{\mu, \boldsymbol{\square}, a}\left(S_{\mathbf{\bullet}}^{\mu}\right)^{a} \sum_{i=1}^{N} \frac{\left(J_{\mathbf{\square}}^{i}\right)^{a}}{\sqrt{N}}\left(S_{\mathbf{\square}(i)}^{\mu}\right)^{a}\right]\right\rangle_{J^{i}}\right\rangle_{S^{\mu}} \\
& =\left\langle 1+\sum_{\mu} \sum_{\mathbf{\square}} \sum_{a} i \eta_{\mu, \boldsymbol{\square}, a}\left(S_{\mathbf{\bullet}}^{\mu}\right)^{a} \sum_{i=1}^{N} \frac{\left\langle\left(J_{\mathbf{\square}}^{i}\right)^{a}\right\rangle_{J^{i}}}{\sqrt{N}}\left(S_{\mathbf{\square}(i)}^{\mu}\right)^{a}\right.
\end{aligned}
$$

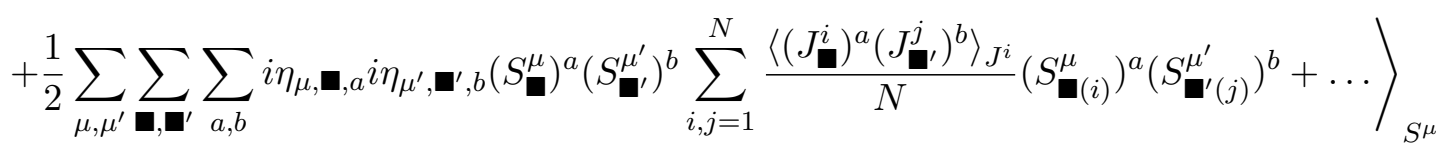

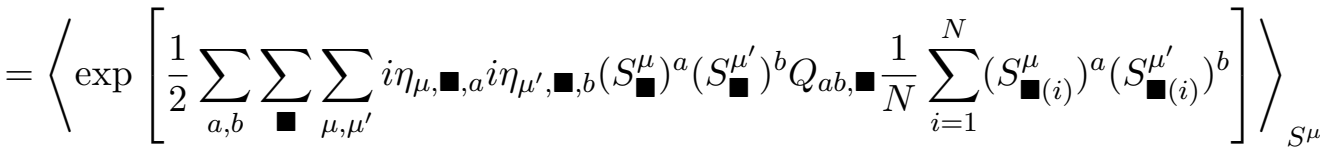

$$
\begin{aligned}
& =\exp \left[\frac{1}{2} \sum_{a, b} \sum_{\square} \sum_{\mu} i \eta_{\mu, \boldsymbol{\varpi}, a} i \eta_{\mu, \mathbf{\square}, b} q_{a b, \boldsymbol{\square}} Q_{a b, \boldsymbol{\varpi}} \frac{1}{N} \sum_{i=1}^{N} q_{a b, \boldsymbol{\square}(i)}+\ldots\right]
\end{aligned}
$$

Here we see that higher orders of cumulants ... with respect to the average $\langle\cdots\rangle_{S^{\mu}}$ cannot be neglected. In the present paper we neglect the higher order terms, which can be considered as a kind of Gaussian approximation for the fluctuation of the spins. 


\section{A.2.2 Modified model}

For the modified model introduced in sec. 2.3 we find,

$$
\begin{aligned}
& \left\langle\prod_{\mu, \mathbf{\square}, a} \exp \left[i \eta_{\mu, \boldsymbol{\varpi}, a} \sum_{i=1}^{N} \frac{\left(J_{\mathbf{\square}}^{i}\right)^{a}}{\sqrt{N}} \sum_{\nu=1}^{M} \frac{\xi_{\mu \nu}}{\sqrt{M}}\left(S_{\mathbf{\bullet}}^{\nu}\right)^{a}\left(S_{\mathbf{\square}(i)}^{\nu}\right)^{a}\right]\right\rangle_{S^{\mu}, J^{i}, \xi_{\mu \nu}} \\
& =\left\langle 1+\sum_{\mu} \sum_{\mathbf{\square}} \sum_{a} i \eta_{\mu, \boldsymbol{\varpi}, a} \sum_{i=1}^{N} \frac{\left(J_{\mathbf{\square}}^{i}\right)^{a}}{\sqrt{N}} \sum_{\nu=1}^{M} \frac{\xi_{\mu \nu}}{\sqrt{M}}\left(S_{\mathbf{\square}}^{\nu}\right)^{a}\left(S_{\mathbf{\square}(i)}^{\nu}\right)^{a}\right. \\
& \left.+\frac{1}{2} \sum_{\mu, \mu^{\prime}} \sum_{\mathbf{\square}, \mathbf{\square}^{\prime}} \sum_{a, b} i \eta_{\mu, \mathbf{\square}, a} i \eta_{\mu^{\prime}, \mathbf{\varpi}^{\prime}, b} \sum_{i, j=1}^{N} \frac{\left(J_{\mathbf{\square}}^{i}\right)^{a}\left(J_{\mathbf{\square}^{\prime}}^{j}\right)^{b}}{N} \sum_{\nu, \nu^{\prime}=1}^{M} \frac{\xi_{\mu \nu} \xi_{\mu^{\prime} \nu^{\prime}}}{M}\left(S_{\mathbf{\square}}^{\nu}\right)^{a}\left(S_{\mathbf{\square}^{\prime}}^{\nu^{\prime}}\right)^{b}\left(S_{\mathbf{\square}(i)}^{\nu}\right)^{a}\left(S_{\mathbf{\square}^{\prime}(j)}^{\nu^{\prime}}\right)^{b}+\ldots\right\rangle_{S^{\mu}, J^{i}, \xi_{\mu \nu}} \\
& =\left\langle\exp \left[\frac{1}{2} \sum_{a, b} \sum_{\mathbf{\square}} \sum_{\mu} i \eta_{\mu, \boldsymbol{\varpi}, a} i \eta_{\mu, \boldsymbol{\varpi}, b} \frac{1}{N} \sum_{i=1}^{N}\left\langle\left(J_{\mathbf{\square}}^{i}\right)^{a}\left(J_{\mathbf{\bullet}}^{i}\right)^{b}\right\rangle_{J^{i}} \frac{1}{M} \sum_{\nu=1}^{M}\left(S_{\mathbf{\square}}^{\nu}\right)^{a}\left(S_{\mathbf{\square}}^{\nu}\right)^{b}\left(S_{\mathbf{\square}}^{\nu}(i)\right)^{a}\left(S_{\mathbf{\square}(i)}^{\nu}\right)^{b}\right]\right\rangle_{S^{\mu}} \\
& =\prod_{\mu, \varpi} \exp \left[\sum_{a, b} i \eta_{\mu, \boldsymbol{\varpi}, a} i \eta_{\mu, \boldsymbol{\varpi}, b} q_{a b, \boldsymbol{\square}} Q_{a b, \boldsymbol{\varpi}} \frac{1}{N} \sum_{i=1}^{N} q_{a b, \boldsymbol{\varpi}(i)}\right]
\end{aligned}
$$

Here we can see that the higher order terms in Eq. (67) are absent.

\section{A.3 Total free-energy}

Now we can write

$$
\begin{aligned}
& V^{n}\left(\mathbf{S}_{0}, \mathbf{S}_{L}\right)=\int \prod_{a<b}\left(\prod_{\square} d Q_{a b, \boldsymbol{\varpi}} e^{c S_{\text {ent,bond }}[\hat{Q} \mathbf{\square}]} \prod_{\square \backslash \text { output }} d q_{a b, \boldsymbol{\varpi}} e^{M S_{\text {ent,spin }}[\hat{q} \mathbf{\square}]}\right) \\
& \prod_{\mathbf{\square}}\left\{\left(\prod_{a} \int \frac{d \eta_{a}}{\sqrt{2 \pi}} W_{\eta_{a}}\right) e^{-\frac{1}{2} \sum_{a, b} \eta_{a} \eta_{b} Q_{a b, \boldsymbol{m}} q_{a b, \boldsymbol{\bullet}} \frac{\sum_{i=1}^{N} q_{a b, \mathbf{m}(i)}}{N}}\right\}^{M} \\
& =\int \prod_{a<b}\left(\prod_{\square} d Q_{a b, \boldsymbol{\varpi}} e^{c S_{\text {ent,bond }}[\hat{Q} \mathbf{\square}]} \prod_{\square \backslash \text { output }} d q_{a b, \boldsymbol{\varpi}} e^{M S_{\text {ent }, \text { spin }}[\hat{q} \varpi]}\right)
\end{aligned}
$$

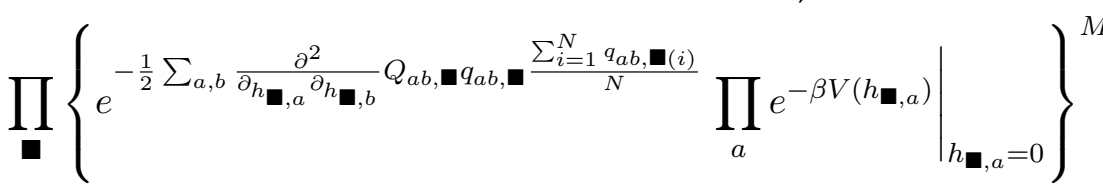

Given the structure of the network, it is natural to assume that the saddle point values only depend on the layer $l=0,1,2, \ldots, L$, as Eq. (14),

$$
Q_{a b, \boldsymbol{\varpi}}^{*}=Q_{a b}(l) \quad q_{a b, \boldsymbol{\varpi}}^{*}=q_{a b}(l)
$$

Then we find,

$$
S_{n}[\{\hat{Q}(l), \hat{q}(l)\}]=\frac{1}{\alpha} \sum_{l=1}^{L} S_{\text {ent,bond }}[\hat{Q}(l)]+\sum_{l=1}^{L-1} S_{\text {ent,spin }}[\hat{q}(l)]+\sum_{l=1}^{L} \mathcal{F}_{\text {int }}[\hat{q}(l-1), \hat{Q}(l), \hat{q}(l)]
$$


with

$$
\mathcal{F}_{\text {int }}[\hat{q}(l-1), \hat{Q}(l), \hat{q}(l)]=\left.\ln e^{\frac{1}{2} \sum_{a b} q_{a b}(l-1) Q_{a b}(l) q_{a b}(l) \partial_{h_{l, a}} \partial_{h_{l, b}}} \prod_{a=1}^{n} e^{-\beta V\left(h_{l, a}\right)}\right|_{h_{l, a}=0}
$$

\section{B RSB solution for the random inputs/outputs}

Here we derive the RSB solution using the Parisi's ansatz explained in sec. 3.3.1.

\section{B.1 Entropic part of the free-energy}

\section{B.1.1 Entropic part of the free-energy due to 'bonds'}

In the $k$-RSB ansatz, the entropic part of the free-energy Eq. (16) due to the 'bonds' can be evaluated as follows. We find $[17,36]$,

$$
\begin{aligned}
\ln \operatorname{det} \hat{Q} & =\ln \left(1+\sum_{j=0}^{k}\left(m_{j}-m_{j+1}\right) Q_{j}\right) \\
& +n \sum_{i=0}^{k}\left(\frac{1}{m_{i+1}}-\frac{1}{m_{i}}\right) \ln \left(1+\sum_{j=i}^{k}\left(m_{j}-m_{j+1}\right) Q_{j}-m_{i} Q_{i}\right)
\end{aligned}
$$

Remembering that $m_{0}=n$ we find,

$$
\begin{aligned}
\left.\partial_{n} S_{\text {ent,bond }}[\hat{Q}]\right|_{n=0}=\left.\frac{1}{2} \partial_{n} \ln \operatorname{det} \hat{Q}\right|_{n=0} & =\frac{1}{2} \frac{Q_{0}}{G_{0}}+\frac{1}{2} \frac{1}{m_{1}} \ln G_{0} \\
& +\frac{1}{2} \sum_{i=1}^{k}\left(\frac{1}{m_{i+1}}-\frac{1}{m_{i}}\right) \ln G_{i}
\end{aligned}
$$

with

$$
G_{i}=1+\sum_{j=i}^{k}\left(m_{j}-m_{j+1}\right) Q_{j}-m_{i} Q_{i} \quad i=0,1, \ldots, k
$$

which implies

$$
Q_{i}=1-G_{k}+\sum_{j=i+1}^{k} \frac{1}{m_{j}}\left(G_{j}-G_{j-1}\right) \quad i=0,1, \ldots, k
$$




\section{B.1.2 Entropic part of the free-energy due to 'spins'}

In the $k$-RSB ansatz, the entropic part of the free-energy Eq. (17) due to the 'spins' can be evaluated as follows

$$
\begin{aligned}
S_{\text {ent }, \text { spin }}[\hat{\epsilon}, \hat{q}]= & \frac{n}{2} \sum_{i=0}^{k} \epsilon_{i} q_{i}\left(m_{i}-m_{i+1}\right)+\frac{n}{2} \epsilon_{k} \\
& +\left.\ln \prod_{i=0}^{k} \exp \left[\frac{\Lambda_{i}^{\text {Ising }}}{2} \sum_{a, b=1}^{n} I_{a b}^{m_{i}} \frac{\partial^{2}}{\partial h_{a} \partial h_{b}}\right] \prod_{a=1}^{n}\left(2 \cosh \left(h_{a}\right)\right)\right|_{\left\{h_{a}=0\right\}}
\end{aligned}
$$

which implies

$$
\begin{aligned}
\left.\partial_{n} S_{\text {ent,spin }}[\hat{\epsilon}, \hat{q}]\right|_{n=0} & =\frac{1}{2} \sum_{i=0}^{k} \epsilon_{i} q_{i}\left(m_{i}-m_{i+1}\right)+\frac{\epsilon_{k}}{2}-f_{\text {Ising }}\left(m_{0}=0,0\right) \\
& =\frac{1}{2} \sum_{i=0}^{k} \epsilon_{i} q_{i}\left(m_{i}-m_{i+1}\right)+\frac{\epsilon_{k}}{2}-\int D z_{0} f_{\text {Ising }}\left(m_{1}, \sqrt{\Lambda_{0}^{\text {Ising }}} z_{0}\right)
\end{aligned}
$$

where $\epsilon_{i}$ 's must be fixed through saddle point equations with respect to variations of them (see below).

In the last two equation of Eq. (79) we used a family of functions which can be obtained recursively as follows [37]. Using

$$
\Lambda_{i}^{\text {Ising }}=\left\{\begin{array}{cc}
-\epsilon_{0} & (i=0) \\
-\epsilon_{i}+\epsilon_{i-1} & (i=1,2, \ldots, k)
\end{array}\right.
$$

we introduce a family of functions defined recursively for $i=0,1,2, \ldots, k$,

$$
\begin{aligned}
e^{-m_{i} f_{\text {Ising }}\left(m_{i}, h\right)} & =e^{\frac{\Lambda_{i}^{\text {Ising }}}{2} \frac{\partial^{2}}{\partial h^{2}}} e^{-m_{i} f_{\text {Ising }}\left(m_{i+1}, h\right)} \\
& =\gamma_{\Lambda_{i}^{\text {Ising }}} \otimes e^{-m_{i} f\left(m_{i+1}, h\right)} \\
& =\int D z_{i} e^{-m_{i} f_{\text {Ising }}\left(m_{i+1}, h-\sqrt{\Lambda_{i}^{\text {Ising }}} z_{i}\right)}
\end{aligned}
$$

with the initial condition

$$
f_{\text {Ising }}\left(m_{k+1}, h\right)=-\ln 2 \cosh (h) .
$$

Here we used an identity

$$
\exp \left(\frac{a}{2} \frac{\partial^{2}}{\partial h^{2}}\right) A(h)=\gamma_{a} \otimes A(h)
$$

and the following short hand notations: $\gamma_{a}(x)$ is a Gaussian

$$
\gamma_{a}(x)=\frac{1}{\sqrt{2 \pi a}} e^{-\frac{x^{2}}{2 a}}
$$


by which we write a convolution of a function $A(x)$ with the Gaussian as,

$$
\gamma_{a} \otimes A(x) \equiv \int d y \frac{e^{-\frac{y^{2}}{2 a}}}{\sqrt{2 \pi a}} A(x-y)=\int \mathcal{D} z A(x-\sqrt{a} z)
$$

where

$$
\int \mathcal{D} z \ldots \equiv \int d z \frac{e^{-\frac{z^{2}}{2}}}{\sqrt{2 \pi}} \cdots
$$

The saddle point equation with respect to variations of $\hat{\epsilon}$ Eq. (18) becomes in the $k$-RSB ansatz, for $i=0,1,2, \ldots, k$,

$$
\begin{aligned}
q_{i} & =\frac{2}{m_{i}-m_{i+1}}\left[\left(-\frac{\partial}{\partial \epsilon_{i}}\right)\left(-f_{\text {Ising }}\left(m_{0}=0,0\right)\right)-\frac{1}{2} \delta_{i k}\right] \\
& =\int d h P_{\text {Ising }}\left(m_{i}, h\right)\left(-f_{\text {Ising }}^{\prime}\left(m_{i}, h\right)\right)^{2}
\end{aligned}
$$

where $f_{\text {Ising }}^{\prime}(m, h)=\partial_{h} f_{\text {Ising }}(m, h)$ and we used (see [17] appendix C)

$$
\left(-\frac{\partial}{\partial \epsilon_{i}}\right)\left(-f_{\text {Ising }}\left(m_{0}=0,0\right)\right)=\frac{1}{2}\left(m_{i}-m_{i+1}\right) \int d h P_{\text {Ising }}\left(m_{i}, h\right)\left(-f_{\text {Ising }}^{\prime}\left(m_{i+1}, h\right)\right)^{2}+\frac{1}{2} \delta_{i, k}
$$

with

$$
P_{\text {Ising }}\left(m_{i}, h\right) \equiv \frac{\delta f_{\text {Ising }}\left(m_{0}, 0\right)}{\delta f_{\text {Ising }}\left(m_{i+1}, h\right)}
$$

which follows a recursion formula (see [17] sec. 8.3.1),

$$
P_{\text {Ising }}\left(m_{j}, h\right)=e^{-m_{j} f_{\text {Ising }}\left(m_{j+1}, h\right)} \gamma_{\Lambda_{j}^{\text {Ising }}} \otimes_{h} \frac{P_{\text {Ising }}\left(m_{j-1}, h\right)}{e^{-m_{j} f_{\text {Ising }}\left(m_{j}, h\right)}} \quad j=1,2, \ldots, k
$$

with the 'boundary condition'

$$
P_{\text {Ising }}\left(m_{0}, h\right)=\frac{1}{\sqrt{2 \pi \Lambda_{0}^{\text {Ising }}}} e^{-\frac{h^{2}}{2 \Lambda_{0}}}
$$

In Eq. $(90) \otimes_{h}$ stands for a convolution with respect to the variable $h$.

The derivatives $f_{\text {Ising }}^{\prime}(m, h)=\partial_{h} f_{\text {Ising }}(m, h)$ can also be obtained recursively. From Eq. (81) and Eq. (82) we find,

$$
f_{\text {Ising }}^{\prime}\left(m_{i}, h\right)=e^{m_{i} f_{\text {Ising }}\left(m_{i}, h\right)} \gamma_{\Lambda_{i}^{\text {Ising }}} \otimes f_{\text {Ising }}^{\prime}\left(m_{i+1}, h\right) e^{-m_{i} f_{\text {Ising }}\left(m_{i+1}, h\right)}
$$

for $i=1,2, \ldots, k$ with the 'boundary condition',

$$
f_{\text {Ising }}^{\prime}\left(m_{k+1}, h\right)=-\tanh (h)
$$




\section{B.2 Interaction part of the free-energy}

The interaction part of the free-energy Eq. (19) becomes in the $k$-RSB ansatz,

$$
\begin{aligned}
\left.\partial_{n} \mathcal{F}_{\text {int }}[\hat{q}(l-1), \hat{Q}(l), \hat{q}(l)]\right|_{n=0} & =\left.\left.\partial_{n} \ln \prod_{i=0}^{k+1} \exp \left[\frac{\Lambda_{i}(l)}{2} \sum_{a, b=1}^{n} I_{a b}^{m_{i}} \frac{\partial^{2}}{\partial h_{a} \partial h_{b}}\right] \prod_{a=1}^{n} e^{-\beta V\left(r\left(h_{a}\right)\right)}\right|_{\left\{h_{a}=0\right\}}\right|_{n=0} \\
& =f\left(m_{0}=0,0, l\right) \\
& =\left.\int D z_{0} f\left(m_{1}, h-\sqrt{\Lambda_{0}}, l\right)\right|_{h=0}
\end{aligned}
$$

with, for $l=1,2, \ldots, L$,

$$
\Lambda(l)=\left\{\begin{array}{cc}
\lambda_{0}(l) & (i=0) \\
\lambda_{i}(l)-\lambda_{i-1}(l) & (i=1,2, \ldots, k+1)
\end{array}\right.
$$

and

$$
\lambda_{i}(l)=q_{i}(l-1) Q_{i}(l) q_{i}(l)
$$

We introduced a family of functions defined recursively for $i=0,1,2, \ldots, k$ and $l=$ $1,2, \ldots, L$,

$$
\begin{aligned}
e^{-m_{i} f\left(m_{i}, h, l\right)} & =e^{\frac{\Lambda_{i}(l)}{2} \frac{\partial^{2}}{\partial h^{2}}} e^{-m_{i} f\left(m_{i+1}, h, l\right)} \\
& =\int D z_{i} e^{-m_{i} f\left(m_{i+1}, h-\sqrt{\Lambda_{i}(l)} z_{i}, l\right)}
\end{aligned}
$$

with the initial condition

$$
f\left(m_{k+1}, l\right)=-\ln \gamma_{\Lambda_{k+1}(l)} \otimes e^{-\beta V(h)}=-\ln \int D z_{k+1} e^{-\beta V\left(h-\sqrt{\Lambda_{k+1}(l)} z_{k+1}\right)}
$$

For the hard-core potential Eq. (6) we find,

$$
f\left(m_{k+1}, h, l\right)=-\ln \Theta\left(\frac{h}{\sqrt{2 \Lambda_{k+1}(l)}}\right)
$$

where

$$
\Theta(x)=\int_{-\infty}^{x} \frac{d y}{\sqrt{\pi}} e^{-y^{2}}
$$

\section{B.3 Saddle point equations}

\section{B.3.1 Variation of $q_{i}(l)^{\prime}$ s}

The saddle point equations Eq. (21) becomes, for $i=0,1,2, \ldots, k$ and $l=1,2, \ldots, L-1$,

$$
\begin{aligned}
0 & =\left.\frac{\partial}{\partial q_{i}(l)} \partial_{n} S_{n}^{\text {ent,spin }}[\hat{q}, \hat{Q}]\right|_{n=0} \\
& =\frac{1}{2} \epsilon_{i}(l)\left(m_{i}-m_{i+1}\right)-\frac{\partial}{\partial q_{i}(l)} \sum_{l^{\prime}=1}^{L} \mathcal{F}_{\text {int }}\left[\hat{q}\left(l^{\prime}-1\right), \hat{Q}\left(l^{\prime}\right), \hat{q}\left(l^{\prime}\right)\right] \\
& =\frac{1}{2} \epsilon_{i}(l)\left(m_{i}-m_{i+1}\right)+\sum_{l^{\prime}=1}^{L} \frac{\partial \lambda_{i}\left(l^{\prime}\right)}{\partial q_{i}(l)}\left(-\frac{\partial f\left(m_{0}=0,0, l^{\prime}\right.}{\partial \lambda_{i}\left(l^{\prime}\right)}\right)
\end{aligned}
$$


from which we find,

$$
\begin{aligned}
\epsilon_{i}(l) & =-\sum_{l^{\prime}=1}^{L} \frac{\partial \lambda_{i}\left(l^{\prime}\right)}{\partial q_{i}(l)} \kappa_{i}\left(l^{\prime}\right) \\
& =-q_{i}(l-1) Q_{i}(l) \kappa_{i}(l)-Q_{i}(l+1) q_{i}(l+1) \kappa_{i}(l+1)
\end{aligned}
$$

where we introduced, for $i=0,1,2, \ldots, k$ and $l=1,2, \ldots, L$,

$$
\kappa_{i}(l) \equiv \int d h P\left(m_{i}, h, l\right)\left(-f^{\prime}\left(m_{i+1}, h, l\right)\right)^{2}
$$

with

$$
P\left(m_{i}, h, l\right) \equiv \frac{\delta f\left(m_{0}, 0, l\right)}{\delta f\left(m_{i+1}, h, l\right)}
$$

which follows a recursion formula (see [17] sec. 8.3.1),

$$
P\left(m_{j}, h, l\right)=e^{-m_{j} f\left(m_{j+1}, h, l\right)} \gamma_{\Lambda_{j}} \otimes_{h} \frac{P\left(m_{j-1}, h, l\right)}{e^{-m_{j} f\left(m_{j}, h, l\right)}} \quad j=1,2, \ldots, k+1
$$

with the 'boundary condition'

$$
P\left(m_{0}, h, l\right)=\frac{1}{\sqrt{2 \pi \Lambda_{0}(l)}} e^{-\frac{h^{2}}{2 \Lambda_{0}(l)}}
$$

The last equation of Eq. (101) is obtained using the following (see [17] appendix C)

$$
\frac{\partial}{\partial \lambda_{i}(l)}\left(-f\left(m_{0}=0,0, l\right)\right)=\frac{1}{2}\left(m_{i}-m_{i+1}\right) \int d h P\left(m_{i}, h, l\right)\left(-f^{\prime}\left(m_{i+1}, h, l\right)\right)^{2}
$$

The derivatives $f^{\prime}(m, h, l)=\partial_{h} f(m, h, l)$ can also be obtained recursively. From Eq. (97) and Eq. (98) we find,

$$
f^{\prime}\left(m_{i}, h, l\right)=e^{m_{i} f\left(m_{i}, h, l\right)} \gamma_{\Lambda_{i}(l)} \otimes f^{\prime}\left(m_{i+1}, h, l\right) e^{-m_{i} f\left(m_{i+1}, h, l\right)}
$$

for $i=1,2, \ldots, k$ with the 'boundary condition',

$$
f^{\prime}\left(m_{k+1}, h, l\right)=-\frac{\int D z_{k+1}\left(e^{-\beta V\left(h-\sqrt{\Lambda_{k+1}(l)}\right)}\right)^{\prime}}{\int D z_{k+1} e^{-\beta V\left(h-\sqrt{\Lambda_{k+1}(l)}\right)}}
$$

which becomes for the hardcore potential (using Eq. (99) and Eq. (100)),

$$
f^{\prime}\left(m_{k+1}, h, l\right)=-\frac{1}{\Theta\left(\frac{h}{\sqrt{2 \Lambda_{k+1}(l)}}\right)} \frac{1}{\sqrt{2 \pi \Lambda_{k+1}(l)}} \exp \left(-\frac{h^{2}}{2 \Lambda_{k+1}(l)}\right)
$$




\section{B.3.2 Variation of $G_{i}(l)$ 's}

For the saddle point equations Eq. (20) it is convenient to consider instead, for $i=0,1,2, \ldots, k$ and $l=1,2, \ldots, L$,

$$
0=\left.\frac{\partial}{\partial G_{i}(l)} \partial_{n} S[\hat{q}, \hat{Q}]\right|_{n=0}
$$

where $G_{i}(l)$ 's are defined in Eq. (76). We obtain (see [17] sec. 8.4),

$$
\begin{aligned}
\frac{Q_{0}(l)}{G_{0}^{2}(l)} & =\alpha q_{0}(l) q_{0}(l-1) \kappa_{0}(l) \\
\frac{1}{G_{i}(l)}-\frac{1}{G_{0}(l)} & =\alpha\left(\sum_{j=0}^{i-1}\left(m_{j}-m_{j+1}\right) q_{j}(l) q_{j}(l-1) \kappa_{j}(l)+m_{i} q_{i}(l) q_{i}(l-1) \kappa_{i}(l)\right) \quad(i=1,2, \ldots, k)(112)
\end{aligned}
$$

\section{B.3.3 Procedure to solve the saddle point equations}

The saddle point equations for a generic finite $k$-RSB ansatz with some fixed values of $0<$ $m_{1}<m_{2}<\ldots<m_{k}<1$ can be solved numerically as follows.

0. Choose a boundary condition by fixing $q_{i}(0)$ and $q_{i}(L)$ for $i=0,1,2, \ldots, k$.

1. Make some guess for the initial values of $q_{i}(l)(l=1,2, \ldots, L-1)$ and $Q_{i}(l)(l=$ $1,2, \ldots, L)$ for $i=0,1,2, \ldots, k$. Then compute $G_{i}(l)$ for $i=0,1, \ldots, k$ and $l=$ $1,2, \ldots, L-1$ using Eq. (76).

2. Do the following (1)-(8) for $l=1,2, \ldots, L$. (1) Compute $\lambda_{i}(l)$ for $i=0,1,2, \ldots, k$ and using Eq. (96). (2) Compute $\Lambda_{i}(l)$ for $i=0,1,2, \ldots, k+1$ using Eq. (95). (3) Compute functions $f\left(m_{i}, h, l\right)$ recursively for $i=k, k-1, \ldots, 0$ using Eq. (97) with the boundary condition given by Eq. (98) (which is Eq. (99) for the hardcore potential). (4) Compute also the derivatives $f^{\prime}\left(m_{i}, h, l\right)$ recursively for $i=k, k-1, \ldots, 2,1$ using Eq. (108) with the boundary condition given by Eq. (109) (which is Eq. (110) for the hardcore potential). (5) Compute functions $P\left(m_{i}, h, l\right)$ recursively for $i=1, \ldots, k$ using Eq. (105) with the boundary condition given by Eq. (106). (6) Compute $\kappa_{i}(l)$ for $i=0,1, \ldots, k$ using Eq. (103). (7) Compute using $G_{i}(l)$ for $i=0,1, \ldots, k$ Eq. (112). (8) Compute $Q_{i}(l)$ for $i=0,1, \ldots, k$ using Eq. (77).

3. Do the following (1)-(6) for $l=1,2, \ldots, L-1$. (1) Compute $\epsilon_{i}(l)$ for $i=0,1,2, \ldots, k$ using Eq. (102). (2) Compute $\Lambda_{i}^{\text {Ising }}(l)$ for $i=0,1,2, \ldots, k$ using Eq. (80). (3) Compute functions $f_{\text {Ising }}\left(m_{i}, h, l\right)$ recursively for $i=k, k-1, \ldots, 0$ using Eq. (81) with the boundary condition given by Eq. (82). (4) Compute also the derivatives $f_{\text {Ising }}^{\prime}\left(m_{i}, h, l\right)$ recursively for $i=k, k-1, \ldots, 2,1$ using Eq. (92) with the boundary condition given by Eq. (93). (5) Compute functions $P_{\text {Ising }}\left(m_{i}, h, l\right)$ recursively for $i=1, \ldots, k$ using Eq. (90) with the boundary condition given by Eq. (91). (6) Compute $q_{i}(l)$ for $i=0,1, \ldots, k$ using Eq. (18).

4. Return to 2 .

The above procedure 1.-4. must be repeated until the solution converges. 


\section{RSB solution for the teacher-student setting}

Here we derive the RSB solution using the Parisi's ansatz explained in sec. 3.3.2.

\section{C.1 Entropic part of the free-energy}

\section{C.1.1 Entropic part of the free-energy due to 'bonds'}

Within the ansatz for the teacher-student setting, the entropic part of the free-energy Eq. (16) due to the 'bonds' can be evaluated as follows. First we find,

$$
\operatorname{det} \hat{Q}^{1+s}=\operatorname{det}\left(\hat{Q}^{s}-R^{2}\right)
$$

Thus we find

$$
\begin{aligned}
\left.\partial_{s} S_{\text {ent,bond }}\left[\hat{Q}^{1+s}\right]\right|_{s=0} & =\left.\frac{1}{2} \partial_{s} \ln \operatorname{det}\left(\hat{Q}^{s}-R^{2}\right)\right|_{s=0} \\
& =\frac{1}{2} \frac{Q_{0}-R^{2}}{G_{0}}+\frac{1}{2} \frac{1}{m_{1}} \ln G_{0} \\
& +\frac{1}{2} \sum_{i=1}^{k}\left(\frac{1}{m_{i+1}}-\frac{1}{m_{i}}\right) \ln G_{i}
\end{aligned}
$$

with

$$
G_{i}=1+\sum_{j=i}^{k}\left(m_{j}-m_{j+1}\right) Q_{j}-m_{i} Q_{i}+R^{2} \quad i=0,1, \ldots, k
$$

which implies

$$
Q_{i}=R^{2}+1-G_{k}+\sum_{j=i+1}^{k} \frac{1}{m_{j}}\left(G_{j}-G_{j-1}\right) \quad i=0,1, \ldots, k
$$

Note that above equations slightly modify Eq. (76) and Eq. (77).

\section{C.1.2 Entropic part of the free-energy due to 'spins'}

Within the same ansatz, the entropic part of the free-energy Eq. (17) due to the 'spins' can be evaluated as follows,

$$
\begin{gathered}
S_{\text {ent,spin }}\left[\hat{\epsilon}^{1+s}, \hat{q}^{1+s}\right]=\frac{s}{2} \epsilon_{r} r+\frac{1}{2} \epsilon_{r}+\frac{s}{2} \sum_{i=0}^{k} \epsilon_{i} q_{i}\left(m_{i}-m_{i+1}\right)+\frac{s}{2} \epsilon_{k} \\
+\left.\ln \exp \left[\frac{\Lambda_{\text {com }}^{\text {Ising }}}{2} \sum_{a, b=0}^{s} \frac{\partial^{2}}{\partial h_{a} \partial h_{b}}\right] \prod_{i=0}^{k} \exp \left[\frac{\Lambda_{i}^{\text {Ising }}}{2} \sum_{a, b=1}^{s} I_{a b}^{m_{i}} \frac{\partial^{2}}{\partial h_{a} \partial h_{b}}\right] \prod_{a=0}^{s}\left(2 \cosh \left(h_{a}\right)\right)\right|_{\left\{h_{a}=0\right\}} \\
=\frac{s}{2} \epsilon_{r} r+\frac{1}{2} \epsilon_{r}+\frac{s}{2} \sum_{i=0}^{k} \epsilon_{i} q_{i}\left(m_{i}-m_{i+1}\right)+\frac{s}{2} \epsilon_{k} \\
+\ln \gamma_{\Lambda_{\text {com }}} \otimes\left(\left.2 \cosh (h) \gamma_{\Lambda_{0}^{\text {Ising }}} \otimes e^{-s f^{\text {Ising }}\left(m_{1}, h\right)}\right|_{h=0}\right.
\end{gathered}
$$


where $\epsilon_{r}$ and $\epsilon_{i}$ 's must be fixed through saddle point equations with respect to variations of them (see below).

In Eq. (117) $I_{a b}^{m_{i}}$ is defined similarly as those used in Eq. (27)-Eq. (29) (see Fig. 2) but with size $s \times s$ instead of $n \times n$. We have also introduced,

$$
\begin{aligned}
& \Lambda_{\mathrm{com}}^{\mathrm{Ising}}=-\epsilon_{r} \\
& \Lambda_{i}^{\mathrm{Ising}}=\left\{\begin{array}{cc}
-\epsilon_{0}+\epsilon_{r} & (i=0) \\
-\epsilon_{i}+\epsilon_{i-1} & (i=1,2, \ldots, k)
\end{array}\right.
\end{aligned}
$$

and used the family of functions defined recursively for $i=0,1,2, \ldots, k$ using Eq. (81) and the initial condition Eq. (82). One just keep in mind that $\Lambda_{0}^{\text {Ising }}$ in Eq. (118) is shifted with respect to that in Eq. (80) due to $\epsilon_{r}$.

The saddle point equations with respect to variations of $\hat{\epsilon}^{1+s}$ Eq. (18) yield $q_{i}$ 's and $r$. Variation with respect to $\epsilon_{i}$ yields the equation for the $q_{i}$ 's, which is formally the same as Eq. (119),

$$
q_{i}=\int d h P_{\text {Ising }}\left(m_{i}, h\right)\left(-f_{\text {Ising }}^{\prime}\left(m_{i}, h\right)\right)^{2}
$$

where $P_{\text {Ising }}\left(m_{i}, h\right)$ can be obtained from the equation Eq. (90). However the initial condition is modified from Eq. (91) to

$$
P_{\text {Ising }}\left(m_{0}, h\right)=\frac{\int D z_{\mathrm{com}} 2 \cosh \left(\sqrt{\Lambda_{\mathrm{com}}^{\text {Ising }}} z_{\mathrm{com}}\right) \frac{1}{\sqrt{2 \pi \Lambda_{0}^{\text {Ising }}}} e^{-\frac{\left(h-\sqrt{\Lambda_{\mathrm{com}}} z_{\mathrm{com}}\right)^{2}}{2 \Lambda_{0}^{\text {Ising }}}}}{\int D z_{\mathrm{com}} 2 \cosh \left(\sqrt{\Lambda_{\mathrm{com}}^{\text {Ising }}} z_{\mathrm{com}}\right)}
$$

Variation with respect to $\epsilon_{r}$ yields the equation for the $r$ as the following. Using

$$
\begin{aligned}
& 0=\frac{\partial}{\partial \epsilon_{r}} S_{\text {ent }, \text { spin }}\left[\hat{\epsilon}, \hat{q}^{1+s}\right]=\frac{1}{2} s r+\frac{1}{2}-\frac{1}{2} \\
& -s \frac{\int D z_{\mathrm{com}} 2 \sinh \left(\sqrt{\Lambda_{\mathrm{com}}} z_{\mathrm{com}}\right) \int D z_{0}\left(-f_{\text {Ising }}^{\prime}\left(m_{1}, \sqrt{\Lambda_{\mathrm{com}}} z_{\mathrm{com}}+\sqrt{\Lambda_{0}^{\mathrm{Ising}}} z_{0}\right)\right)}{\int D z_{\mathrm{com}} 2 \cosh \left(\sqrt{\Lambda_{\mathrm{com}}} z_{\mathrm{com}}\right)}+O\left(s^{2}\right)
\end{aligned}
$$

Thus we find

$$
r=\frac{\int D z_{\mathrm{com}} 2 \sinh \left(\sqrt{\Lambda_{\mathrm{com}}} z_{\mathrm{com}}\right) \int D z_{0}\left(-f_{\text {Ising }}^{\prime}\left(m_{1}, \sqrt{\Lambda_{\mathrm{com}}} z_{\mathrm{com}}+\sqrt{\Lambda_{0}^{\mathrm{Ising}}} z_{0}\right)\right)}{\int D z_{\mathrm{com}} 2 \cosh \left(\sqrt{\Lambda_{\mathrm{com}}} z_{\mathrm{com}}\right)}
$$




\section{C.2 Interaction part of the free-energy}

Within the same ansatz, the interaction part of the free-energy Eq. (19) becomes,

$$
\begin{gathered}
\left.\partial_{s} \mathcal{F}_{\text {int }}\left[\hat{q}^{1+s}(l-1), \hat{Q}^{1+s}(l), \hat{q}^{1+s}(l)\right]\right|_{s=0}=\partial_{s} \ln \exp \left[\frac{\Lambda_{\text {com }}(l)}{2} \sum_{a, b=0}^{s} \frac{\partial^{2}}{\partial h_{a} \partial h_{b}}\right] \exp \left[\frac{\Lambda_{\text {teacher }}(l)}{2} \frac{\partial^{2}}{\partial h_{0}^{2}}\right] \\
\left.\left.\prod_{i=0}^{k+1} \exp \left[\frac{\Lambda_{i}(l)}{2} \sum_{a, b=1}^{s} I_{a b}^{m_{i}} \frac{\partial^{2}}{\partial h_{a} \partial h_{b}}\right] \prod_{a=0}^{s} e^{-\beta V\left(r\left(h_{a}\right)\right)}\right|_{\left\{h_{a}=0\right\}}\right|_{s=0} \\
=\int D z_{\text {com }} \int D z_{\text {teacher }} e^{-\beta V\left(\sqrt{\Lambda_{\text {com }}(l)} z_{\text {com }}+\sqrt{\Lambda_{\text {teacher }}(l)} z_{\text {teacher }}\right)} \\
\int D z_{0} f\left(m_{1}, \sqrt{\Lambda_{\text {com }}(l)} z_{\text {com }}+\sqrt{\Lambda_{0}(l)} z_{0}\right)
\end{gathered}
$$

with, for $l=1,2, \ldots, L$. Here we introduced,

$$
\begin{aligned}
& \Lambda_{\text {com }}(l)=r(l-1) R(l) r(l) \\
& \Lambda_{\text {teacher }}(l)=1-r(l-1) R(l) r(l) \\
& \Lambda_{i}(l)=\left\{\begin{array}{cc}
\lambda_{0}(l)-\Lambda_{\text {com }}(l) & (i=0) \\
\lambda_{i}(l)-\lambda_{i-1}(l) & (i=1,2, \ldots, k+1)
\end{array}\right.
\end{aligned}
$$

with $\lambda_{i}(l)$ 's defined in Eq. (96) which reads as,

$$
\lambda_{i}(l)=q_{i}(l-1) Q_{i}(l) q_{i}(l)
$$

We also used the family of functions $f\left(m_{i}, h\right)$ defined recursively for $i=0,1,2, \ldots, k$ and $l=1,2, \ldots, L$ by Eq. (97) with the initial condition Eq. (98). Note that $\Lambda_{0}$ is shifted with respect to that in Eq. (95) due to $R$ and $r$.

\section{C.3 Saddle point equations}

\section{C.3.1 Variation of $q_{i}(l)$ 's}

For the saddle point equations Eq. (21), we find formally the same result as Eq. (102) which reads as,

$$
\epsilon_{i}(l)=-q_{i}(l-1) Q_{i}(l) \kappa_{i}(l)-Q_{i}(l+1) q_{i}(l+1) \kappa_{i}(l+1)
$$

with $\kappa_{i}$ defined as Eq. (103) which reads as,

$$
\kappa_{i}(l) \equiv \int d h P\left(m_{i}, h, l\right)\left(-f^{\prime}\left(m_{i+1}, h, l\right)\right)^{2} .
$$

The function $P\left(m_{i}, h, l\right)$ can be also be obtained by the same equations as before Eq. (105) but with the initial condition Eq. (106) modified as,

$$
P\left(m_{0}, h, l\right)=\frac{\int D z_{\text {com }} \int D z_{\text {teacher }} e^{-\beta V\left(\sqrt{\Lambda_{\text {com }}(l)} z_{\text {com }}+\sqrt{\Lambda_{\text {teacher }}(l)} z_{\text {teacher }}\right)} \frac{1}{\sqrt{2 \pi \Lambda_{0}(l)}} e^{-\frac{\left(h-\sqrt{\Lambda_{\text {com }}(l)} z_{\text {com }}\right)^{2}}{2 \Lambda_{0}(l)}}}{\int D z_{\text {com }} \int D z_{\text {teacher }} e^{-\beta V\left(\sqrt{\Lambda_{\text {com }}(l)} z_{\text {com }}+\sqrt{\Lambda_{\text {teacher }}(l)} z_{\text {teacher }}\right)}}
$$


Note also that $\Lambda_{0}$ is shifted as in Eq. (124). For the hardcore potential Eq. (6) we find,

$$
P\left(m_{0}, h, l\right)=\frac{1}{\int D z \Theta\left(\frac{\sqrt{\Lambda_{\text {com }}(l)} z}{\sqrt{2\left(\Lambda_{\text {teacher }}(l)\right)}}\right)} \int D z \Theta\left(\frac{\sqrt{\Lambda_{\text {com }}(l) z}}{\sqrt{\left.2 \Lambda_{\text {teacher }}(l)\right)}}\right) \frac{1}{\sqrt{2 \pi \Lambda_{0}(l)}} e^{-\frac{\left(h-\sqrt{\Lambda_{\text {com }}(l)} z\right)^{2}}{2 \Lambda_{0}(l)}}
$$

with $\Theta(h)$ defined in Eq. (100).

\section{C.3.2 Variation of $G_{i}(l)$ 's}

For the saddle point equations Eq. (20), we just need to modify slightly Eq. (112), with $G_{i}$ 's defined in Eq. (115),

$$
\begin{aligned}
& \frac{Q_{0}(l)-R^{2}(l)}{G_{0}^{2}(l)}=\alpha q_{0}(l) q_{0}(l-1) \kappa_{0}(l) \\
& \frac{1}{G_{i}(l)}-\frac{1}{G_{0}(l)}=\alpha\left(\sum_{j=0}^{i-1}\left(m_{j}-m_{j+1}\right) q_{j}(l) q_{j}(l-1) \kappa_{j}(l)+m_{i} q_{i}(l) q_{i}(l-1) \kappa_{i}(l)\right) \quad(i=1,2, \ldots, k)
\end{aligned}
$$

for $l=1,2, \ldots, L$.

\section{C.3.3 Variation of $r$}

Here we consider variation of the free-energy Eq. (24) (see also Eq. (15)) with respect to $r(l)$, , for $l=1,2, \ldots, L-1$,

$$
\begin{aligned}
0 & =\left.\frac{\partial}{\partial r(l)} \partial_{s} S_{1+s}[\{\hat{Q}(l), \hat{q}(l)\}]\right|_{s=0} \\
& =\left.\frac{\partial}{\partial r(l)} \frac{1}{\alpha} \partial_{s} S_{\text {ent,spin }}\left[\hat{q}^{1+s}(l)\right]\right|_{s=0}-\left.\frac{\partial}{\partial r(l)} \sum_{l^{\prime}=1}^{L} \partial_{s} \mathcal{F}_{\text {int }}\left[\hat{q}^{1+s}\left(l^{\prime}-1\right), \hat{Q}^{1+s}\left(l^{\prime}\right), \hat{q}^{1+s}\left(l^{\prime}\right)\right]\right|_{s=0}(131
\end{aligned}
$$

Variation of the entropic part (spin) of the free-energy Eq. (117) yields,

$$
\left.\frac{\partial}{\partial r} \partial_{s} S_{\text {ent }, \text { spin }}\left[\hat{q}^{1+s}\right]\right|_{s=0}=\frac{1}{2} \epsilon_{r}
$$

On the other hand, variation of the interaction part of the free-energy Eq. (123) yields, for $l=1,2, \ldots, L-1$,

$$
\begin{aligned}
& -\left.\frac{\partial}{\partial r(l)} \sum_{l^{\prime}=1}^{L} \partial_{s} \mathcal{F}_{\text {int }}\left[\hat{q}^{1+s}(l-1), \hat{Q}^{1+s}(l), \hat{q}^{1+s}(l)\right]\right|_{s=0} \\
& =-\sum_{l^{\prime}=1}^{L}\left(\frac{\partial \Lambda_{\text {com }}\left(l^{\prime}\right)}{\partial r(l)} \frac{\partial}{\partial \Lambda_{\text {com }}\left(l^{\prime}\right)}+\frac{\partial \Lambda_{\text {teacher }}\left(l^{\prime}\right)}{\partial r(l)} \frac{\partial}{\partial \Lambda_{\text {teacher }}\left(l^{\prime}\right)}+\frac{\partial \Lambda_{0}\left(l^{\prime}\right)}{\partial r(l)} \frac{\partial}{\partial \Lambda_{0}\left(l^{\prime}\right)}\right) \\
& \left.\partial_{s} \mathcal{F}_{\text {int }}\left[\hat{q}^{1+s}(l-1), \hat{Q}^{1+s}(l), \hat{q}^{1+s}(l)\right]\right|_{s=0} \\
& =\frac{1}{2} r(l-1) R(l) \kappa_{\text {inter }}(l)+\frac{1}{2} R(l+1) r(l+1) \kappa_{\text {inter }}(l+1)
\end{aligned}
$$


where we introduced

$\kappa_{\text {inter }}(l) \equiv \frac{\int D z_{\text {com }} g_{\text {teacher }}^{\prime}\left(\sqrt{\Lambda_{\text {com }}(l)} z_{\text {com }}, l\right) \int D z_{0}\left(-f^{\prime}\left(m_{1}, \sqrt{\Lambda_{\text {com }}(l)} z_{\text {com }}+\sqrt{\Lambda_{0}(l)} z_{0}\right)\right)}{\int D z_{\text {com }} g_{\text {teacher }}\left(\sqrt{\Lambda_{\text {com }}(l)} z_{\text {com }}\right)}$

with

$$
g_{\text {teacher }}(h) \equiv \int D z_{\text {teacher }} e^{-\beta V\left(h-\sqrt{\Lambda_{\text {teacher }}} z_{\text {teacher }}\right)}
$$

For the hardcore potential Eq. (6), $g_{\text {teacher }}(h)=\Theta\left(h / \sqrt{2 \Lambda_{\text {teacher }}}\right)$ with $\Theta(h)$ defined in Eq. (100) and $g_{\text {teacher }}^{\prime}(h)=e^{-h^{2} / 2 \Lambda_{\text {teacher }}} / \sqrt{2 \pi \Lambda_{\text {teacher }}}$.

Using the above results we find, for $l=1,2, \ldots, L-1$,

$$
\epsilon_{r}(l)=-r(l-1) R(l) \kappa_{\text {inter }}(l)-R(l+1) r(l+1) \kappa_{\text {inter }}(l+1)
$$

\section{C.3.4 Variation of $R$}

Finally we consider variation of the free-energy Eq. (24) (see also Eq. (15)) with respect to $R(l)$, for $l=1,2, \ldots, L$,

$$
\begin{aligned}
0 & =\left.\frac{\partial}{\partial R(l)} \partial_{s} S_{1+s}[\{\hat{Q}(l), \hat{q}(l)\}]\right|_{s=0} \\
& =\left.\frac{\partial}{\partial R(l)} \frac{1}{\alpha} \partial_{s} S_{\text {ent,bond }}\left[\hat{Q}^{1+s}(l)\right]\right|_{s=0}-\left.\frac{\partial}{\partial R(l)} \mathcal{F}_{\text {int }}\left[\left\{\hat{Q}^{1+s}(l), \hat{q}^{1+s}(l)\right\}\right]\right|_{s=0}
\end{aligned}
$$

Variation of the entropic part (bond) of the free-energy Eq. (114) yields,

$$
\left.\frac{\partial}{\partial R} \partial_{s} S_{\text {ent,bond }}\left[\hat{Q}^{1+s}\right]\right|_{s=0}=R\left\{-\frac{Q_{0}-R^{2}}{G_{0}^{2}}+\sum_{i=1}^{k}\left(\frac{1}{m_{i+1}}-\frac{1}{m_{i}}\right)\left(\frac{1}{G_{i}}-\frac{1}{G_{0}}\right)\right\}
$$

On the other hand, variation of the interaction part of the free-energy Eq. (123) yields,

$$
\begin{aligned}
& \left.\frac{\partial}{\partial R(l)} \partial_{s} \mathcal{F}_{\text {int }}\left[\hat{q}^{1+s}(l-1), \hat{Q}^{1+s}(l), \hat{q}^{1+s}(l)\right]\right|_{s=0} \\
& =\left(\frac{\partial \Lambda_{\text {com }}(l)}{\partial R(l)} \frac{\partial}{\partial \Lambda_{\text {com }}(l)}+\frac{\partial \Lambda_{\text {teacher }}(l)}{\partial R(l)} \frac{\partial}{\partial \Lambda_{\text {teacher }}(l)}+\frac{\partial \Lambda_{0}(l)}{\partial R(l)} \frac{\partial}{\partial \Lambda_{0}(l)}\right) \\
& \left.\quad \partial_{s} \mathcal{F}_{\text {int }}\left[\hat{q}^{1+s}(l-1), \hat{Q}^{1+s}(l), \hat{q}^{1+s}(l)\right]\right|_{s=0} \\
& =\frac{1}{2} r(l-1) r(l) \kappa_{\text {inter }}(l)
\end{aligned}
$$

with $\kappa_{\text {inter }}(l)$ defined in Eq. (134).

Using these results in Eq. (137) we find,

$$
0=\frac{1}{\alpha} R(l)\left\{-\frac{Q_{0}-R^{2}}{G_{0}^{2}}+\sum_{i=1}^{k}\left(\frac{1}{m_{i+1}}-\frac{1}{m_{i}}\right)\left(\frac{1}{G_{i}}-\frac{1}{G_{0}}\right)\right\}+\frac{1}{2} r(l-1) r(l) \kappa_{\text {inter }}(l)
$$

